TRANSACTIONS OF THE

AMERICAN MATHEMATICAL SOCIETY

Volume 354, Number 7 , Pages $2775-2796$

S 0002-9947(02)02969-0

Article electronically published on March 6, 2002

\title{
APPLICATIONS OF LANGLANDS' FUNCTORIAL LIFT OF ODD ORTHOGONAL GROUPS
}

\author{
HENRY H. KIM
}

\begin{abstract}
Together with Cogdell, Piatetski-Shapiro and Shahidi, we proved earlier the existence of a weak functorial lift of a generic cuspidal representation of $S O_{2 n+1}$ to $G L_{2 n}$. Recently, Ginzburg, Rallis and Soudry obtained a more precise form of the lift using their integral representation technique, namely, the lift is an isobaric sum of cuspidal representations of $G L_{n_{i}}$ (more precisely, cuspidal representations of $G L_{2 n_{i}}$ such that the exterior square $L$-functions have a pole at $s=1$ ). One purpose of this paper is to give a simpler proof of this fact in the case that a cuspidal representation has one supercuspidal component. In a separate paper, we prove it without any condition using a result on spherical unitary dual due to Barbasch and Moy. We give several applications of the functorial lift: First, we parametrize square integrable representations with generic supercuspidal support, which have been classified by Moeglin and Tadic. Second, we give a criterion for cuspidal reducibility of supercuspidal representations of $G L_{m} \times S O_{2 n+1}$. Third, we obtain a functorial lift from generic cuspidal representations of $\mathrm{SO}_{5}$ to automorphic representations of $G L_{5}$, corresponding to the $L$-group homomorphism $S p_{4}(\mathbb{C}) \longrightarrow G L_{5}(\mathbb{C})$, given by the second fundamental weight.
\end{abstract}

\section{INTRODUCTION}

The purpose of this note is to give several applications of Langlands' functorial lift of odd-orthogonal groups. Recall the $L$-group homomorphism ${ }^{L} S O_{2 n+1}^{\circ}=$ $S p_{2 n}(\mathbb{C}) \hookrightarrow G L_{2 n}(\mathbb{C})={ }^{L} G L_{2 n}^{\circ}$. Langlands' functoriality predicts that there is a map from cuspidal representations of $S O_{2 n+1}\left(\mathbb{A}_{F}\right)$ to automorphic representations of $G L_{2 n}\left(\mathbb{A}_{F}\right)$, where $\mathbb{A}_{F}$ is the ring of adeles of a number field $F$. Throughout this note, cuspidal representations mean unitary ones.

In C-Ki-PS-S, it is proved that given a generic cuspidal representation of $S O_{2 n+1}\left(\mathbb{A}_{F}\right)$, there exists a weak lift (see Definition 2.1 for the notion). We prove here the existence of a strong lift. Especially, a generic cuspidal representation with one supercuspidal component has the strong lift which is of the expected form, namely, if $\pi$ is a generic cuspidal representation of $S O_{2 n+1}\left(\mathbb{A}_{F}\right)$ with one supercuspidal component, then the lift $\Pi$ is an automorphic representation of $G L_{2 n}\left(\mathbb{A}_{F}\right)$, which is of the form Ind $\sigma_{1} \otimes \cdots \otimes \sigma_{p}$, where the $\sigma_{i}$ 's are (unitary) self-contragredient cuspidal representations of $G L_{n_{i}}\left(\mathbb{A}_{F}\right)$. In a separate paper [Ki6], we prove that it is

Received by the editors September 25, 2000 and, in revised form, February 21, 2001 and September 27, 2001.

2000 Mathematics Subject Classification. Primary 22E55, 11F70.

Partially supported by NSF grant DMS9988672, NSF grant DMS9729992 (at IAS) and by Clay Mathematics Institute. 
true without any condition, namely, the lift of any generic cuspidal representation of $S O_{2 n+1}\left(\mathbb{A}_{F}\right)$ is of the form Ind $\sigma_{1} \otimes \cdots \otimes \sigma_{p}$, where the $\sigma_{i}$ 's are (unitary) selfcontragredient cuspidal representations of $G L_{2 n_{i}}\left(\mathbb{A}_{F}\right)$ such that $L\left(s, \sigma_{i}, \wedge^{2}\right)$ has a pole at $s=1$. In order to do so, we need a deep result on spherical unitary dual due to Barbasch and Moy $[\mathrm{B}-\mathrm{Mo}$. One purpose of this paper is to give a simpler proof in the case that a cuspidal representation has one supercuspidal component. Recently Ginzburg, Rallis and Soudry also proved that the lift is of the above form without any condition, using their integral representation technique. They can also obtain the backward lifting.

We give several applications of the functorial lift to local problems. First, we parametrize square integrable representations with generic supercuspidal support, which has been classified by Moeglin and Tadic [M-Ta]. More precisely, let $G=S O_{2 n+1}(k)$, where $k$ is a $p$-adic field of characteristic zero and let $W_{k}$ be the Weil group of $k$. The local Langlands' correspondence predicts that an admissible homomorphism $\phi: W_{k} \times S L_{2}(\mathbb{C}) \longrightarrow{ }^{L} G^{\circ}=S p_{2 n}(\mathbb{C})$, parametrizes a finite set $\Pi_{\phi}$, called $L$-packet, of isomorphism classes of irreducible admissible representations of $G$, and every admissible irreducible representation of $G$ belongs to $\Pi_{\phi}$ for a unique $\phi$. Since the local Langlands' correspondence is available for $G L_{n}(k)[\mathrm{H}-\mathrm{T}]$, [He , we will use the $L$-group homomorphism ${ }^{L} S O_{2 n+1}^{\circ}=S p_{2 n}(\mathbb{C}) \hookrightarrow G L_{2 n}(\mathbb{C})={ }^{L} G L_{2 n}^{\circ}$, to obtain the parametrization for square integrable representations with generic supercuspidal support. We should note that given an admissible homomorphism $\phi: W_{k} \times S L_{2}(\mathbb{C}) \longrightarrow{ }^{L} G^{\circ}=S p_{2 n}(\mathbb{C})$, which parametrizes a non-supercuspidal square integrable representation with generic supercuspidal support, the $L$-packet $\Pi_{\phi}$ contains other representations besides the non-supercuspidal square integrable representations with generic supercuspidal support. We speculate that the remaining ones are non-generic supercuspidal representations and non-supercuspidal square integrable representations with non-generic supercuspidal support. Also for the parametrization, we need an assumption (Assumption 5.1) that the local Artin exterior square (symmetric square, resp.) $L$-function has a pole at $s=0$ if and only if the local Shahidi's exterior square (symmetric square, resp.) $L$-function has a pole at $s=0$. This assumption is the same as the assertion that the Shahidi's $L$ functions are Artin $L$-functions. It may be even more difficult to show the equality of their $\epsilon$-factors.

Second, we give a criterion for cuspidal reducibility of supercuspidal representations of $G L_{m} \times S O_{2 n+1}$. More precisely, let $\rho$ be a self-contragredient supercuspidal representation of $G L_{m}(k)$ and $\tau$ be a generic supercuspidal representation of $S O_{2 n+1}(k)$. Then there exists a unique $s_{0} \geq 0$ such that the induced representation Ind $|\operatorname{det}|^{s} \rho \otimes \tau$ is reducible at $s=s_{0},-s_{0}$ and irreducible at all other points. The deep result of Shahidi [Sh1] is that $s_{0} \in\left\{0, \frac{1}{2}, 1\right\}$. We say that $(\rho, \tau)$ satisfies $(C i)$ if Ind $|\operatorname{det}|^{s} \rho \otimes \tau$ is reducible at $s=i$. We give a precise criterion of when $(\rho, \tau)$ satisfies $(C i)$ in terms of the functorial lift of $\tau$.

Third, we obtain a functorial lift from cuspidal representations of $S_{5}\left(\mathbb{A}_{F}\right)$ to automorphic representations of $G L_{5}\left(\mathbb{A}_{F}\right)$, corresponding to the $L$-group homomorphism $S p_{4}(\mathbb{C}) \longrightarrow G L_{5}(\mathbb{C})$, given by the second fundamental weight: Given a generic cuspidal representation $\pi$ of $S O_{5}\left(\mathbb{A}_{F}\right)$, first we obtain a strong lift $\Pi$ to $G L_{4}\left(\mathbb{A}_{F}\right)$. In [Ki4], we showed that the exterior square lift $\bigwedge^{2} \Pi$ is an automorphic representation of $G L_{6}\left(\mathbb{A}_{F}\right)$. We show that $\bigwedge^{2} \Pi=\tau \boxplus 1$, where $\tau$ is an automorphic representation of $G L_{5}\left(\mathbb{A}_{F}\right) ; \tau$ is the desired functorial lift. 
Acknowledgments. I would like to thank the Institute for Advanced Study for inviting me to participate in the special year in the Theory of Automorphic Forms and $L$-functions, particularly the organizers of the special year, E. Bombieri, H. Iwaniec, R.P. Langlands, and P. Sarnak. I would like to thank Professor J. Cogdell for many discussions on the topic of this paper. I am extremely grateful for the referee for many comments in improving this paper.

\section{Strong LifT From $S O_{2 n+1}$ TO $G L_{2 n}$}

Throughout this paper, let $F$ be a number field and $\mathbb{A}_{F}$ be the ring of adeles. We fix an additive character $\psi=\bigotimes_{v} \psi_{v}$ of $\mathbb{A}_{F} / F$.

Definition 2.1. Let $\pi=\bigotimes_{v} \pi_{v}$ be a generic cuspidal representation of $S O_{2 n+1}\left(\mathbb{A}_{F}\right)$. We say that an automorphic representation $\Pi=\bigotimes_{v} \Pi_{v}$ of $G L_{2 n}\left(\mathbb{A}_{F}\right)$ is a strong lift of $\pi$ if every $\Pi_{v}$ is a lift of $\pi_{v}$ for all $v$, in the sense that

$$
\begin{aligned}
\gamma\left(s, \sigma_{v} \times \pi_{v}, \psi_{v}\right) & =\gamma\left(s, \sigma_{v} \times \Pi_{v}, \psi_{v}\right), \\
L\left(s, \sigma_{v} \times \pi_{v}\right) & =L\left(s, \sigma_{v} \times \Pi_{v}\right),
\end{aligned}
$$

for all generic irreducible representation $\sigma_{v}$ of $G L_{m}\left(F_{v}\right), 1 \leq m \leq 2 n-1$. Here the left-hand side is the $\gamma$-factor and L-factor defined in [Sh1, section 7] and the righthand side is the one defined in [J-PS-S]. Due to local Langlands' correspondence, they are the Artin $\gamma$ - and L-factors.

If the above equality holds for almost all $v$, then $\Pi$ is called the weak lift of $\pi$.

Recall the equalities

$$
\begin{aligned}
& \gamma\left(s, \sigma_{v} \times \pi_{v}, \psi_{v}\right)=\epsilon\left(s, \sigma_{v} \times \pi_{v}, \psi_{v}\right) \frac{L\left(1-s, \tilde{\sigma}_{v} \times \tilde{\pi}_{v}\right)}{L\left(s, \sigma_{v} \times \pi_{v}\right)}, \\
& \gamma\left(s, \sigma_{v} \times \Pi_{v}, \psi_{v}\right)=\epsilon\left(s, \sigma_{v} \times \Pi_{v}, \psi_{v}\right) \frac{L\left(1-s, \tilde{\sigma}_{v} \times \tilde{\Pi}_{v}\right)}{L\left(s, \sigma_{v} \times \Pi_{v}\right)} .
\end{aligned}
$$

Hence the equalities of $\gamma$ - and $L$-factors imply the equality of $\epsilon$-factors. Note that if $\Pi_{v}$ is generic for all $v$, the above identities uniquely determine $\Pi$. Recall how $L$ - and $\epsilon$-factors are defined from [Sh1, section 7]. From the theory of local coefficients, which is defined through intertwining operators, a $\gamma$-factor $\gamma\left(s, \pi_{v}, r_{i}, \psi_{v}\right)$ is defined for every irreducible generic admissible representation $\pi_{v}$ and certain finite dimensional representation $r_{i}$. If $\pi_{v}$ is tempered, $L\left(s, \pi_{v}, r_{i}\right)$ is defined to be

$$
L\left(s, \pi_{v}, r_{i}\right)=P_{\pi_{v}, i}\left(q_{v}^{-s}\right)^{-1},
$$

where $P_{\pi_{v}, i}$ is the unique polynomial satisfying $P_{\pi_{v}, i}(0)=1$ such that $P_{\pi_{v}, i}\left(q_{v}^{-s}\right)$ is the numerator of $\gamma\left(s, \pi_{v}, r_{i}, \psi_{v}\right)$. We define the $\epsilon$-factor using the identity

$$
\gamma\left(s, \pi_{v}, r_{i}, \psi_{v}\right)=\epsilon\left(s, \pi_{v}, r_{i}, \psi_{v}\right) \frac{L\left(1-s, \tilde{\pi}_{v}, r_{i}\right)}{L\left(s, \pi_{v}, r_{i}\right)} .
$$

Hence if $\pi_{v}$ is tempered, then the $\gamma$-factor canonically defines both the $L$-factor and the $\epsilon$-factor. If $\pi_{v}$ is non-tempered, write it as a Langlands' quotient of an induced representation formed from a twist of tempered representations (actually by standard module conjecture, $\pi_{v}$ is the full induced representation), and we can write the corresponding intertwining operator as a product of rank-one operators. For these rank-one operators, there correspond $L$-factors and we define $L\left(s, \pi_{v}, r_{i}\right)$ to be the product of these rank-one $L$-factors. We then define $\epsilon$-factor to satisfy the above relation. 
Let $\pi=\bigotimes_{v} \pi_{v}$ be a generic cuspidal representation of $S O_{2 n+1}\left(\mathbb{A}_{F}\right)$. Recall

Theorem 2.2 ([C-Ki-PS-S $]$ ). There exists a weak lift $\Pi=\bigotimes_{v} \Pi_{v}$. It is an automorphic representation of $G L_{2 n}\left(\mathbb{A}_{F}\right)$ and if $\pi_{v}$ is unramified, $\Pi_{v}$ is generic. Moreover, $\Pi_{v}$ is a lift of $\pi_{v}$ for all $v=\infty$ and any finite place $v$ where $\pi_{v}$ is unramified.

We want to show that there exists a strong lift. In order to prove the existence of a strong lift, first we need to construct a local lift for all $v$. Then we apply the converse theorem twice. For the converse theorem and its applications to the lifting, we will freely use the fundamental paper [C-Ki-PS-S].

First, we show that a generic supercuspidal representation of $S O_{2 n+1}\left(F_{v}\right)$ has a local lift to $G L_{2 n}\left(F_{v}\right)$, which is tempered.

We use the following setup. Let $k$ be a local $p$-adic field of characteristic zero. Let $\rho$ be a generic supercuspidal representation of $S_{2 n+1}(k)$. Then by [Sh1, Proposition 5.1], there exists a number field $F$ and a generic cuspidal automorphic representation $\pi=\bigotimes_{w} \pi_{w}$ of $S O_{2 n+1}\left(\mathbb{A}_{F}\right)$ such that $F_{v}=k, \pi_{v}=\rho$ and $\pi_{w}$ is unramified for all $w<\infty$ and $w \neq v$.

Let $\Pi$ be a weak lift of $\pi$ such that $\Pi_{w}$ is a lift of $\pi_{w}$ for $w \neq v$. By the classification of automorphic representations [J-S], it is equivalent to a subquotient of

$$
\Xi=\operatorname{Ind}|\operatorname{det}|^{r_{1}} \sigma_{1} \otimes \cdots \otimes|\operatorname{det}|^{r_{p}} \sigma_{p}
$$

where the $\sigma_{i}$ 's are unitary cuspidal representations of $G L_{n_{i}}\left(\mathbb{A}_{F}\right)$. Then $\Pi_{v}$ is a subquotient of $\Xi_{v}$.

Lemma 2.3. $\left|r_{i}\right|<\frac{1}{2}, i=1, \ldots, p$.

Proof. Consider an unramified local component $\pi_{w}$ of $S O_{2 n+1}\left(F_{w}\right)$. Since it is generic and unitary, it has the form

$$
\pi_{w}=\text { Ind }||^{a_{1}} \mu_{1} \otimes \cdots \otimes||^{a_{n}} \mu_{n},
$$

where the $\mu_{1}, \ldots, \mu_{n}$ 's are unramified unitary characters of $F_{w}^{\times}$and $0 \leq a_{n} \leq \cdots \leq$ $a_{1}<\frac{1}{2}$ (see $\left[\mathrm{Yo}\right.$, Theorem B]). Hence the lift $\Pi_{w}$ is of the form

$$
\Pi_{w}=\text { Ind }||^{a_{1}} \mu_{1} \otimes \cdots \otimes||^{a_{n}} \mu_{n} \otimes||^{-a_{n}} \mu_{n}^{-1} \otimes \cdots \otimes||^{-a_{1}} \mu_{1}^{-1} .
$$

Note that $\Xi_{w}=$ Ind $\mid$ det $\left.\right|^{r_{1}} \sigma_{1 w} \otimes \cdots \otimes|\operatorname{det}|^{r_{p}} \sigma_{p w}$ and $\sigma_{i w}$ is of the form Ind ||$^{b_{1}} \nu_{1} \otimes$ ||$^{b_{2}} \nu_{2} \otimes \cdots \otimes||^{-b_{1}} \nu_{1}$, where $\frac{1}{2}>b_{1} \geq b_{2} \geq \cdots$ and $\nu_{j}$ 's are unitary characters.

Since $\Pi_{w}$ is unramified, it is the unique unramified subquotient of $\Xi_{w}$. Since $\Pi_{w}$ is generic, by Lil, Theorem 2.2], $\Pi_{w}=\Xi_{w}$. Comparing the $a_{i}, b_{j}, r_{l}$ 's, we obtain our result.

Proposition 2.4. $r_{1}=\cdots=r_{p}=0$. Hence $\Pi_{v}=\Xi_{v}$ and it is a local lift of $\pi_{v}$. Moreover, it is tempered.

Proof. Let $\Pi_{v}^{\prime}$ be an another constituent of $\Xi_{v}$ and let $\Pi^{\prime}=\bigotimes_{w \neq v} \Pi_{w} \otimes \Pi_{v}^{\prime}$. Then by the result of Langlands [La3], it is an automorphic representation of $G L_{2 n}\left(\mathbb{A}_{F}\right)$ which is a weak lift of $\pi$. We first show that, for discrete series $\sigma_{v}$ of $G L_{m}\left(F_{v}\right.$ ) (any $m)$,

$$
\gamma\left(s, \sigma_{v} \times \pi_{v}, \psi_{v}\right)=\gamma\left(s, \sigma_{v} \times \Pi_{v}, \psi_{v}\right)=\gamma\left(s, \sigma_{v} \times \Pi_{v}^{\prime}, \psi_{v}\right) .
$$


By $\left[\mathrm{Ro}\right.$, we can find a cuspidal representation $\sigma$ of $G L_{m}\left(\mathbb{A}_{F}\right)$ whose local component at $v$ is $\sigma_{v}$. Consider the three Rankin-Selberg $L$-functions $L(s, \sigma \times \pi)$, $L(s, \sigma \times \Pi)$ and $L\left(s, \sigma \times \Pi^{\prime}\right)$. All three have the functional equations:

$$
\begin{aligned}
L(s, \sigma \times \pi) & =\epsilon(s, \sigma \times \pi) L(1-s, \tilde{\sigma} \times \tilde{\pi}), \\
L(s, \sigma \times \Pi) & =\epsilon(s, \sigma \times \Pi) L(1-s, \tilde{\sigma} \times \tilde{\Pi}), \\
L\left(s, \sigma \times \Pi^{\prime}\right) & =\epsilon\left(s, \sigma \times \Pi^{\prime}\right) L\left(1-s, \tilde{\sigma} \times \tilde{\Pi}^{\prime}\right) .
\end{aligned}
$$

From these equalities, we obtain

$$
\gamma\left(s, \sigma_{v} \times \pi_{v}, \psi_{v}\right)=\gamma\left(s, \sigma_{v} \times \Pi_{v}, \psi_{v}\right)
$$

and

$$
\gamma\left(s, \sigma_{v} \times \pi_{v}, \psi_{v}\right)=\gamma\left(s, \sigma_{v} \times \Pi_{v}^{\prime}, \psi_{v}\right) .
$$

We only prove the first equality: Since $\pi_{w}$ is unramified for $w \neq v, w<\infty, \Pi_{w}$ is the lift of $\pi_{w}$ for all $w \neq v$. Hence

$$
L\left(s, \sigma_{w} \times \pi_{w}\right)=L\left(s, \sigma_{w} \times \Pi_{w}\right), \quad \epsilon\left(s, \sigma_{w} \times \pi_{w}, \psi_{w}\right)=\epsilon\left(s, \sigma_{w} \times \Pi_{w}, \psi_{w}\right),
$$

for all $w \neq v$. The functional equations above can be written in the form

$$
\begin{aligned}
\gamma\left(s, \sigma_{v} \times \pi_{v}, \psi_{v}\right) & =\prod_{w \neq v} \frac{L\left(s, \sigma_{w} \times \pi_{w}\right)}{\epsilon\left(s, \sigma_{w} \times \pi_{w}, \psi_{w}\right) L\left(1-s, \tilde{\sigma}_{w} \times \tilde{\pi}_{w}\right)}, \\
\gamma\left(s, \sigma_{w} \times \Pi_{w}, \psi_{w}\right) & =\prod_{w \neq v} \frac{L\left(s, \sigma_{w} \times \Pi_{w}\right)}{\epsilon\left(s, \sigma_{w} \times \Pi_{w}, \psi_{w}\right) L\left(1-s, \tilde{\sigma}_{w} \times \tilde{\Pi}_{w}\right)} .
\end{aligned}
$$

Hence $\gamma\left(s, \sigma_{v} \times \pi_{v}, \psi_{v}\right)=\gamma\left(s, \sigma_{v} \times \Pi_{v}, \psi_{v}\right)$.

We write $\Xi_{v}$ to be in the Langlands' situation and take $\Pi_{v}^{\prime}$ to be the Langlands' quotient of $\Xi_{v}$, namely, the quotient of

$$
\Xi_{v}=\operatorname{Ind}|\operatorname{det}|^{a_{1}} \tau_{1 v} \otimes \cdots \otimes|\operatorname{det}|^{a_{l}} \tau_{l v},
$$

where the $\tau_{i v}$ 's are discrete series of $G L_{m_{i}}\left(F_{v}\right), a_{1} \geq \cdots \geq a_{l}$, and $\left|a_{i}\right|<1$. The last inequality comes from the fact that $\left|r_{i}\right|<\frac{1}{2}$ by Lemma 2.3 and $\sigma_{i v}$ are unitary representations of $G L_{n_{i}}\left(F_{v}\right)$. Suppose one of the $a_{i}$ 's is not zero. Since $\Pi_{v}^{\prime}$ has a trivial central character, $a_{l}<0$.

By $\left[\mathrm{Ro}\right.$, let $\tau_{l}$ be a cuspidal representation of $G L_{m_{i}}\left(\mathbb{A}_{F}\right)$ whose local component at $v$ is $\tau_{l v}$. Then

$$
\gamma\left(s, \tilde{\tau}_{l v} \times \pi_{v}, \psi_{v}\right)=\gamma\left(s, \tilde{\tau}_{l v} \times \Pi_{v}^{\prime}, \psi_{v}\right)=\prod_{i=1}^{l} \gamma\left(s+a_{i}, \tilde{\tau}_{l v} \times \tau_{i v}, \psi_{v}\right) .
$$

In order to proceed, we need to use the fact that $\pi_{v}$ is supercuspidal: Suppose $\tau_{l v}$ is a Steinberg representation, given as the subrepresentation of $\operatorname{Ind}|\operatorname{det}|^{\frac{p-1}{2}} \eta \otimes$ $|\operatorname{det}|^{\frac{p-1}{2}-1} \eta \otimes \cdots \otimes|\operatorname{det}|^{-\frac{p-1}{2}} \eta$. Then the left-hand side has possible poles only at $R e s=\frac{p+1}{2}$, and possible zeros only at $\operatorname{Re} s=-\frac{p-1}{2}$ (see, for example, [Ki7]).

On the other hand, suppose $\tau_{i v}$ is a Steinberg representation, given as the subrepresentation of

$$
\text { Ind }|\operatorname{det}|^{\frac{p_{i}-1}{2}} \eta_{i} \otimes|\operatorname{det}|^{\frac{p_{i}-1}{2}-1} \eta_{i} \otimes \cdots \otimes|\operatorname{det}|^{-\frac{p_{i}-1}{2}} \eta_{i} .
$$

Then (we suppose $\left.p \geq p_{i}\right) \gamma\left(s, \tilde{\tau}_{l v} \times \tau_{i v}, \psi_{v}\right)$ has possible poles at $R e s=$ $\frac{p+1}{2}+\frac{p_{i}+1}{2}-1, \frac{p+1}{2}+\frac{p_{i}+1}{2}-2, \ldots, \frac{p+1}{2}+\frac{p_{i}+1}{2}-p_{i}$; possible zeros at $R e s=$ 
$-\left(\frac{p-1}{2}+\frac{p_{i}-1}{2}\right),-\left(\frac{p-1}{2}+\frac{p_{i}-1}{2}-1\right), \ldots,-\left(\frac{p-1}{2}-\frac{p_{i}-1}{2}\right)$ (if $p \leq p_{i}$, then exchange $p_{i}$ and $p$; see [Ki7]).

Especially, $\gamma\left(s, \tilde{\tau}_{l v} \times \tau_{l v}, \psi_{v}\right)$ has poles at $\operatorname{Re} s=p, p-1, \ldots, 1$ and zeros at $\operatorname{Re} s=-(p-1), \ldots,-1,0$. Consider the pole at Re $s=p$. Since $\left|a_{i}\right|<1$, the only possible way to match poles is when $p=1$, namely, $\tau_{l v}$ is supercuspidal. In that case, $\gamma\left(s+a_{l}, \tilde{\tau}_{l v} \times \tau_{l v}, \psi_{v}\right)$ has poles at Res $=1-a_{l}$ and zeros at $\operatorname{Re} s=-a_{l}$. The poles at $R e s=1-a_{l}$ cannot be cancelled, since $a_{l}<0$. Contradiction.

Hence $a_{i}=0$ for all $i$ and this implies that $r_{1}=\cdots=r_{p}=0$. Therefore, $\Xi_{v}=\operatorname{Ind} \tau_{1 v} \otimes \cdots \otimes \tau_{l v}$, where $\tau_{i v}$ 's are discrete series of $G L_{m_{i}}\left(F_{v}\right)$. By a wellknown result (see, for example, [Ta]), $\Xi_{v}$ is irreducible and is tempered. Hence $\Xi_{v}=\Pi_{v}$.

We can give a more precise form of the lift $\Pi_{v}$.

Lemma 2.5. Let $\pi_{v}$ be a generic supercuspidal representation of $S O_{2 n+1}\left(F_{v}\right)$. Then the lift $\Pi_{v}$ is a tempered representation, of the form $\Pi_{v}=\sigma_{1} \boxplus \cdots \boxplus \sigma_{p}$, where the $\sigma_{i}$ 's are supercuspidal representations of $G L_{n_{i}}\left(F_{v}\right)$ such that $L\left(s, \sigma_{i}, \wedge^{2}\right)$ has a pole at $s=0$. (Here $L\left(s, \sigma_{i}, \wedge^{2}\right)$ is the Shahidi's L-function given by the Langlands-Shahidi method [Sh1]. More precisely, $L\left(s, \sigma, \wedge^{2}\right)$ comes from the theory of Eisenstein series relative to $G L_{2 n} \subset S O_{4 n}$.) In particular, $n_{i}$ is even, and the $\sigma_{i}$ 's are self-contragredient and have the trivial central character. Moreover, the $\sigma_{i}$ 's are inequivalent.

Proof. We showed above that $\Pi_{v}$ is tempered. Any tempered representation of $G L_{n}\left(F_{v}\right)$ is unitarily induced from the discrete series of $G L$, i.e.,

$$
\Pi_{v}=\operatorname{Ind} \sigma_{1} \otimes \cdots \otimes \sigma_{p},
$$

where the $\sigma_{i}$ 's are discrete series of $G L$. Suppose one of them, say $\sigma_{1}$, is not supercuspidal. Then compare the two $L$-functions

$$
L\left(s, \tilde{\sigma}_{1} \times \pi_{v}\right)=L\left(s, \tilde{\sigma}_{1} \times \Pi_{v}\right) .
$$

Suppose $\sigma_{1}$ is given as the subrepresentation of $|\operatorname{det}|^{\frac{a-1}{2}} \rho \otimes \cdots \otimes \mid$ det $\left.\right|^{-\frac{a-1}{2}} \rho$, where $a$ is a positive integer with $a>1$ and $\rho$ is a supercuspidal representation of $G L$. Then

$$
L\left(s, \tilde{\sigma}_{1} \times \Pi_{v}\right)=\prod_{i=1}^{p} L\left(s, \tilde{\sigma}_{1} \times \sigma_{i}\right)
$$

Here $L\left(s, \tilde{\sigma}_{1} \times \sigma_{1}\right)=\prod_{i=0}^{a-1} L(s+i, \tilde{\rho} \times \rho)$ and $L(s, \tilde{\rho} \times \rho)$ has a pole at $s=0$. Since the local $L$-functions have no zeros, $L\left(s, \tilde{\sigma}_{1} \times \Pi_{v}\right)$ has a pole at $s=-(a-1)$. On the other hand, $L\left(s, \tilde{\sigma}_{1} \times \pi_{v}\right)=L\left(s+\frac{a-1}{2}, \tilde{\rho} \times \pi_{v}\right)$. Since $L\left(s, \tilde{\rho} \times \pi_{v}\right)$ has a possible pole only at $\operatorname{Re} s=0, L\left(s, \tilde{\sigma}_{1} \times \pi_{v}\right)$ has no pole at $s=-(a-1)$. We obtain a contradiction.

Hence $\Pi_{v}=$ Ind $\sigma_{1} \otimes \cdots \otimes \sigma_{p}$, where the $\sigma_{i}$ 's are supercuspidal representations of $G L$. If $\sigma_{1}$ is not self-contragredient, then consider

$$
L\left(s, \tilde{\sigma}_{1} \times \pi_{v}\right)=L\left(s, \tilde{\sigma}_{1} \times \Pi_{v}\right)=\prod_{i=1}^{k} L\left(s, \tilde{\sigma}_{1} \times \sigma_{i}\right) .
$$

The left-hand side does not have a pole at $s=0$ [Sh1, Corollary 7.6], while the right-hand side has a pole at $s=0$. Contradiction. In the same way, we show that the $\sigma_{i}$ 's are self-contragredient. 
Now consider two $L$-functions: $L\left(s, \sigma_{1} \times \pi_{v}\right)$ and $L\left(s, \sigma_{1}, S_{y m^{2}}\right)$. (Here $L\left(s, \sigma_{1}, S y m^{2}\right)$ is the Shahidi's $L$-function given by the Langlands-Shahidi method Sh1]. More precisely, $L\left(s, \sigma_{1}, S_{y m^{2}}\right)$ comes from the theory of Eisenstein series relative to $G L_{2 n} \subset S O_{4 n+1}$.)

If we consider the supercuspidal representation $\sigma_{1} \otimes \pi_{v}$ for $M\left(F_{v}\right)=G L_{m}\left(F_{v}\right) \times$ $S O_{2 n+1}\left(F_{v}\right) \subset S O_{2(m+n)+1}\left(F_{v}\right)$, the local coefficient attached to $\left(M, \sigma_{1} \otimes \pi_{v}\right)$ has as its denominator $L\left(s, \sigma_{1} \times \pi_{v}\right) L\left(2 s, \sigma_{1}, S_{y m^{2}}\right)$ (see [Sh1). So by [Sh1, Corollary 7.6], only one of the two $L$-functions can have a simple pole at $s=0$. Since $L\left(s, \sigma_{1} \times \pi_{v}\right)$ has a pole at $s=0, L\left(s, \sigma_{1}, S y m^{2}\right)$ is holomorphic at $s=0$. Consider the identity $L\left(s, \sigma_{1} \times \sigma_{1}\right)=L\left(s, \sigma_{1}, \wedge^{2}\right) L\left(s, \sigma_{1}, S_{y m^{2}}\right)$. Since $\sigma_{1}$ is self-contragredient, the lefthand side has a pole at $s=0$. Hence $L\left(s, \sigma_{1}, \wedge^{2}\right)$ has a pole at $s=0$. So by [Sh1. Lemma 7.4], $n_{1}$ is even. (It comes from the fact that if $n$ is odd, the parabolic subgroup $P=M N, M=G L_{n}$, is not self-conjugate in $S O_{2 n}$.) We prove the same thing for all $\sigma_{i}, i=2, \ldots, p$.

Suppose $\sigma_{1} \simeq \sigma_{2}$. Then $L\left(s, \sigma_{1} \times \pi_{v}\right)$ has a double pole at $s=0$, which is a contradiction (see [Sh1, Corollary 7.6]).

It remains to prove that the central character of the $\sigma_{i}$ 's is trivial. As in Ki6. Proposition 3.8], we use the similitude group $G_{S O} O_{4 n}\left(F_{v}\right)$ [Go], namely,

$$
G S O_{4 n}=\left\{\left.g \in G L_{4 n}\right|^{t} g J_{4 n} g=\lambda(g) J_{4 n}, \quad \operatorname{det}(g) \lambda(g)^{-2 n}=1 ; \lambda(g) \in G L_{1}\right\} .
$$

Let $P=M N$ with $M=G L_{2 n} \times G L_{1}$. Let $\sigma$ be a supercuspidal representation of $G L_{2 n}\left(F_{v}\right)$ with the central character $\omega_{\sigma}$ and let $\chi$ be a unitary character. Then $\sigma \otimes \chi$ is a supercuspidal representation of $M\left(F_{v}\right)$. [G0, 2.8] shows that $w_{0}(\sigma \otimes \chi) \simeq \sigma \otimes \chi$ if and only if $\sigma \simeq \tilde{\sigma}$ and $\omega_{\sigma}=1$. Hence if $\sigma \simeq \tilde{\sigma}$ and $\omega_{\sigma} \neq 1$, then $w_{0}(\sigma \otimes \chi) \nsucceq \sigma \otimes \chi$. The $L$-function which shows up as a normalizing factor of the intertwining operator [Sh1, section 7], is $L\left(s, \sigma, \wedge^{2}\right)$. Hence by [Sh1, Corollary 7.6], we can see that $L\left(s, \sigma, \wedge^{2}\right)$ has no pole at $s=0$.

Next we show that a non-supercuspidal generic square integrable representation of $S O_{2 n+1}\left(F_{v}\right)$ has a lift to $G L_{2 n}\left(F_{v}\right)$. First, we need the following which is wellknown [Z].

Lemma. In the language of [Ca-Sh], any standard module of $G L_{n}\left(F_{v}\right)$ satisfies injectivity, namely, the following induced representation has the unique irreducible subrepresentation which is generic;

$$
\text { Ind }|\operatorname{det}|^{a_{1}} \tau_{1} \otimes \cdots \otimes|\operatorname{det}|^{a_{m}} \tau_{m} \otimes \sigma_{1} \otimes \cdots \otimes \sigma_{l} \otimes|\operatorname{det}|^{-a_{1}} \tilde{\tau}_{1} \otimes \cdots \otimes|\operatorname{det}|^{-a_{m}} \tilde{\tau}_{m},
$$

where $\tau_{1}, \ldots, \tau_{m}, \sigma_{1}, \ldots, \sigma_{l}$ are discrete series of $G L$ and $a_{1} \geq \cdots \geq a_{m}>0$.

Let $\pi_{v}$ be a non-supercuspidal generic square integrable representation of $S O_{2 n+1}\left(F_{v}\right)$. (The discussion below would be true for any non-supercuspidal square integrable representations with generic supercuspidal support, if we assume multiplicativity of the $\gamma$ - and $L$-factors.) Then by the classification of discrete series for odd-orthogonal groups [Ja2], [M-Ta, $\pi_{v}$ is a subrepresentation of an induced representation (see section 5 for more precise parametrization)

$$
\text { Ind }|\operatorname{det}|^{a_{1}} \tau_{1} \otimes \cdots \otimes|\operatorname{det}|^{a_{m}} \tau_{m} \otimes \tau_{0}
$$

where $\tau_{1}, \ldots, \tau_{m}$ are discrete series representations of $G L$ and $\tau_{0}$ is a generic supercuspidal representation of $S O_{2 l+1}\left(F_{v}\right)$. Let $\Pi_{0}$ be the local lift of $\tau_{0}$. By the above lemma, the induced representation

$$
\text { Ind }|\operatorname{det}|^{a_{1}} \tau_{1} \otimes \cdots \otimes|\operatorname{det}|^{a_{m}} \tau_{m} \otimes \Pi_{0} \otimes|\operatorname{det}|^{-a_{1}} \tilde{\tau}_{1} \otimes \cdots \otimes|\operatorname{det}|^{-a_{m}} \tilde{\tau}_{m},
$$


has the unique generic irreducible subrepresentation. We denote it by $\Pi_{v}$. Then

Proposition 2.6. $\Pi_{v}$ is the local lift of $\pi_{v}$. It is unique. Moreover, $\Pi_{v}$ is tempered.

Proof. Let $\sigma_{v}$ be a discrete series of $G L_{p}\left(F_{v}\right)$. Then

$$
\begin{aligned}
\gamma\left(s, \sigma_{v} \times \pi_{v}, \psi_{v}\right) & =\gamma\left(s, \sigma_{v} \times \tau_{0}, \psi_{v}\right) \prod_{i=1}^{m} \gamma\left(s+a_{i}, \sigma_{v} \times \tau_{i}, \psi_{v}\right) \gamma\left(s-a_{i}, \sigma_{v} \times \tilde{\tau}_{i}, \psi_{v}\right) \\
& =\gamma\left(s, \sigma_{v} \times \Pi_{0}, \psi_{v}\right) \prod_{i=1}^{m} \gamma\left(s+a_{i}, \sigma_{v} \times \tau_{i}, \psi_{v}\right) \gamma\left(s-a_{i}, \sigma_{v} \times \tilde{\tau}_{i}, \psi_{v}\right) \\
& =\gamma\left(s, \sigma_{v} \times \Pi_{v}, \psi_{v}\right) .
\end{aligned}
$$

By multiplicativity of $L$-factors ([Sh5, Theorem 5.2]; we note that the assumption in the statement of the theorem has been verified by [Ca-Sh] and $[\mathrm{Mu}$. .), the same equality holds for $L$-factors also, namely,

$$
L\left(s, \sigma_{v} \times \pi_{v}\right)=L\left(s, \sigma_{v} \times \Pi_{v}\right) .
$$

The temperedness of $\Pi_{v}$ follows easily from the above identity, by comparing poles of both sides. More precisely, let $\Pi_{v}$ be a Langlands' quotient of

$$
\text { Ind }|\operatorname{det}|^{a_{1}} \eta_{1} \otimes \cdots \otimes|\operatorname{det}|^{a_{l}} \eta_{l},
$$

where the $\eta_{i}$ 's are discrete series of $G L_{m_{i}}\left(F_{v}\right), a_{1} \geq \cdots \geq a_{l}$. Suppose one of the $a_{i}$ 's is not zero. Since $\Pi_{v}$ has a trivial central character, $a_{l}<0$. Then

$$
L\left(s, \tilde{\eta}_{l} \times \pi_{v}\right)=\prod_{i=1}^{l} L\left(s+a_{i}, \tilde{\eta}_{l} \times \eta_{i}\right) .
$$

The left-hand side has no poles for $\operatorname{Re} s>0$. But the right-hand side has a pole at Re $s=-a_{l}>0$.

Since $\Pi_{v}$ is generic, the equality of $\gamma$ - and $L$-factors implies that it is unique.

Proposition 2.7. Any generic tempered representation of $\mathrm{SO}_{2 n+1}\left(F_{v}\right)$ has the local lift, which is tempered. Hence any generic irreducible representation of $S_{2 n+1}\left(F_{v}\right)$ has a local lift.

Proof. Any tempered representation $\pi_{v}$ of $S O_{2 n+1}\left(F_{v}\right)$ is a subrepresentation of

$$
\text { Ind } \sigma_{1} \otimes \cdots \otimes \sigma_{m} \otimes \tau,
$$

where $\sigma_{1}, \ldots, \sigma_{m}$ are discrete series of $G L$ and $\tau$ is a discrete series of $S O_{2 l+1}\left(F_{v}\right)$. Let $\Pi_{0}$ be the local lift of $\tau$. Then the following induced representation is irreducible (see, for example, [Ta]) and tempered. We denote it by $\Pi_{v}$;

$$
\text { Ind } \sigma_{1} \otimes \cdots \otimes \sigma_{k} \otimes \Pi_{0} \otimes \tilde{\sigma}_{m} \otimes \cdots \otimes \tilde{\sigma}_{1} .
$$

It is easy to show that $\Pi_{v}$ is the local lift of $\pi_{v}$ by showing the equality of $\gamma$-factors from multiplicativity of $\gamma$-factors.

By standard module conjecture, proved by Muić [Mu, Theorem 0.4], any generic irreducible representation $\pi_{v}$ can be written as a full induced representation

$$
\text { Ind }|\operatorname{det}|^{r_{1}} \sigma_{1} \otimes \cdots \otimes|\operatorname{det}|^{r_{m}} \sigma_{m} \otimes \tau,
$$

where $\sigma_{1}, \ldots, \sigma_{m}$ are discrete series of $G L$ and $\tau$ is a tempered representation of $S O_{2 l+1}\left(F_{v}\right)$. Let $\Pi_{0}$ be the local lift of $\tau$ and let $\Pi_{v}$ be the unique quotient of

$$
\Xi_{v}=\operatorname{Ind}|\operatorname{det}|^{r_{1}} \sigma_{1} \otimes \cdots \otimes|\operatorname{det}|^{r_{m}} \sigma_{m} \otimes \Pi_{0} \otimes|\operatorname{det}|^{-r_{m}} \tilde{\sigma}_{m} \otimes \cdots \otimes|\operatorname{det}|^{-r_{1}} \tilde{\sigma}_{1} .
$$


Note that if $r_{i}=\frac{1}{2}$ for some $i, \Xi_{v}$ is reducible and hence $\Pi_{v}$ is not generic. Then we can show from multiplicativity of $\gamma$ - and $L$-factors that for any discrete series $\sigma_{v}$ of $G L$,

$$
\gamma\left(s, \sigma_{v} \times \pi_{v}, \psi_{v}\right)=\gamma\left(s, \sigma_{v} \times \Pi_{v}, \psi_{v}\right), \quad L\left(s, \sigma_{v} \times \pi_{v}\right)=L\left(s, \sigma_{v} \times \Pi_{v}\right) .
$$

Namely, $\Pi_{v}$ is a local lift of $\pi_{v}$.

Remark. Note that in the above, when $\Xi_{v}$ is reducible, it has the unique irreducible subrepresentation $\Pi_{v}^{\prime}$ which is generic. In that case, by multiplicativity of $\gamma$-factors, we have

$$
\gamma\left(s, \sigma_{v} \times \pi_{v}, \psi_{v}\right)=\gamma\left(s, \sigma_{v} \times \Pi_{v}^{\prime}, \psi_{v}\right) .
$$

However, $L\left(s, \sigma_{v} \times \pi_{v}\right) \neq L\left(s, \sigma_{v} \times \Pi_{v}^{\prime}\right)$. Let us give an example. Let $k$ be a $p$ adic field of characteristic zero and $\rho$ be a discrete series of $G L_{2}(k)$ with the trivial central character. Then $\rho$ can be considered a representation of $P G L_{2}(k) \simeq S O_{3}(k)$. Consider $\tau=\operatorname{Ind}_{G L_{2} \times S O_{3}}|\operatorname{det}|^{\frac{1}{2}} \rho \otimes \rho$. Then $\tau$ is irreducible and hence it is a generic representation of $\mathrm{SO}_{7}(k)$. However, its lift $\Pi$ to $G L_{6}(k)$ is not generic. It is the unique quotient of

$$
\operatorname{Ind} d_{G L_{2} \times G L_{2} \times G L_{2}}^{G L_{6}}|\operatorname{det}|^{\frac{1}{2}} \rho \otimes \rho \otimes|\operatorname{det}|^{-\frac{1}{2}} \rho .
$$

Let $\Pi^{\prime}$ be the unique irreducible subrepresentation. Then

$$
\gamma(s, \rho \times \tau, \psi)=\gamma(s, \rho \times \Pi, \psi)=\gamma\left(s, \rho \times \Pi^{\prime}, \psi\right),
$$

but

$$
\begin{aligned}
L(s, \rho \times \tau) & =L\left(s-\frac{1}{2}, \rho \times \rho\right) L(s, \rho \times \rho) L\left(s+\frac{1}{2}, \rho \times \rho\right) ; \\
L\left(s, \rho \times \Pi^{\prime}\right) & =L(s, \rho \times \rho) L\left(s+\frac{1}{2}, \rho \times \rho\right) .
\end{aligned}
$$

Before proceeding, we recall here the converse theorem of Cogdell and PiatetskiShapiro (see [C-Ki-PS-S for details):

Theorem ([Co-PS]). Suppose $\Pi=\bigotimes_{v} \Pi_{v}$ is an irreducible admissible representation of $G L_{N}\left(\mathbb{A}_{F}\right)$ such that $\omega_{\Pi}=\bigotimes_{v} \omega_{\Pi_{v}}$ is a grössencharacter of $F$. Let $S$ be a finite set of finite places and let $\mathcal{T}^{S}(m)$ be a set of cuspidal representations of $G L_{m}\left(\mathbb{A}_{F}\right)$ that are unramified at all places $v \in S$. Suppose $L(s, \sigma \times \Pi)$ is nice (i.e., entire, bounded in vertical strips and satisfies a functional equation) for all cuspidal representations $\sigma \in \mathcal{T}^{S}(m), m<N$. Then there exists an automorphic representation $\Pi^{\prime}$ of $G L_{N}\left(\mathbb{A}_{F}\right)$ such that $\Pi_{v} \simeq \Pi_{v}^{\prime}$ for all $v \notin S$.

Theorem 2.8. Let $\pi=\bigotimes_{v} \pi_{v}$ be a generic cuspidal representation of $S O_{2 n+1}\left(\mathbb{A}_{F}\right)$. Then a strong lift exists. It is an automorphic representation of $G L_{2 n}\left(\mathbb{A}_{F}\right)$.

Proof. Since $\pi$ is generic, each $\pi_{v}$ is generic. For each $\pi_{v}$, we found a local lift $\Pi_{v}$. Let $\Pi=\bigotimes_{v} \Pi_{v}$. It is an irreducible admissible representation of $G L_{2 n}\left(\mathbb{A}_{F}\right)$. Pick two finite places $v_{1}, v_{2}$, where both $\pi_{v_{1}}, \pi_{v_{2}}$ are unramified. Let $S_{i}=\left\{v_{i}\right\}, i=1,2$. We apply the above converse theorem twice to $\Pi$ with $S_{1}$ and $S_{2}$, to obtain two automorphic representations $\Pi_{1}, \Pi_{2}$ of $G L_{2 n}\left(\mathbb{A}_{F}\right)$ such that $\Pi_{1 v} \simeq \Pi_{v}$ for $v \neq v_{1}$, and $\Pi_{2 v} \simeq \Pi_{v}$ for $v \neq v_{2}$. Hence $\Pi_{1 v} \simeq \Pi_{2 v} \simeq \Pi_{v}$ for all $v \neq v_{1}, v_{2}$.

By the classification of automorphic representations [J-S], $\Pi_{1}$ and $\Pi_{2}$ are equivalent to subquotients of $\Xi_{1}, \Xi_{2}$, resp. which are of the form

$$
\text { Ind }|\operatorname{det}|^{r_{1}} \sigma_{1} \otimes \cdots \otimes|\operatorname{det}|^{r_{m}} \sigma_{m} \text {, }
$$


where the $\sigma_{i}$ 's are (unitary) cuspidal representations of $G L_{n_{i}}\left(\mathbb{A}_{F}\right)$.

If $\pi_{v}, v \neq v_{1}, v_{2}$ is unramified, $\Pi_{1 v}, \Pi_{2 v}$ are the unique unramified subquotient of $\Xi_{1 v}, \Xi_{2 v}$, resp. But since $\Pi_{1 v} \simeq \Pi_{2 v} \simeq \Pi_{v}$ is generic by [Lil Theorem 2.2], $\Xi_{1 v} \simeq \Pi_{1 v} \simeq \Pi_{2 v} \simeq \Xi_{2 v}$ if $\pi_{v}$ is unramified and $v \neq v_{1}, v_{2}$. Hence by strong multiplicity one theorem $\mathrm{J}-\mathrm{S}$ ], $\Xi_{1 w} \simeq \Xi_{2 w}$ for all $w$. Especially, $\Xi_{1 v_{i}} \simeq \Xi_{2 v_{i}}$ for $i=1,2$. Since $\pi_{v_{i}}, i=1,2$, is unramified, $\Pi_{1 v_{i}} \simeq \Xi_{1 v_{i}}$ and $\Pi_{2 v_{i}} \simeq \Xi_{2 v_{i}}$ for $i=1,2$. Hence $\Xi_{1 v_{i}}$ and $\Xi_{2 v_{i}}$ are irreducible, and $\Pi_{1 v} \simeq \Xi_{1 v} \simeq \Xi_{2 v} \simeq \Pi_{2 v} \simeq \Pi_{v}$ for $v=v_{1}, v_{2}$. Therefore $\Pi \simeq \Pi_{1} \simeq \Pi_{2}$. This proves that $\Pi=\bigotimes_{v} \Pi_{v}$ is an automorphic representation of $G L_{2 n}\left(\mathbb{A}_{F}\right)$.

If $\pi=\bigotimes_{v} \pi_{v}$ has one supercuspidal component, one has a more precise form of the lift.

Theorem 2.9. Let $\pi=\bigotimes_{v} \pi_{v}$ be a generic cuspidal representation of $S O_{2 n+1}\left(\mathbb{A}_{F}\right)$ such that $\pi_{v_{0}}$ is supercuspidal. Then the strong lift exists and it is of the form $\sigma_{1} \boxplus \cdots \boxplus \sigma_{k}$, where $\sigma_{i}$ is a self-contragredient cuspidal representation of $G L_{n_{i}}\left(\mathbb{A}_{F}\right)$.

Proof. Let $\Pi$ be a strong lift of $\pi$, constructed in Theorem 2.8. It is equivalent to a subquotient of

$$
\Xi=\operatorname{Ind}|\operatorname{det}|^{r_{1}} \sigma_{1} \otimes \cdots \otimes|\operatorname{det}|^{r_{p}} \sigma_{p}
$$

where the $\sigma_{i}$ 's are (unitary) cuspidal representations of $G L_{n_{i}}\left(\mathbb{A}_{F}\right)$. In particular, $\Pi_{v_{0}}$ is a subquotient of $\Xi_{v_{0}}$. By repeating the arguments of Lemma 2.3 and Proposition 2.4, we can show that $r_{1}=\cdots=r_{p}=0$ and $\Xi_{v_{0}}=\Pi_{v_{0}}$ is tempered. Hence $\Xi=$ Ind $\sigma_{1} \otimes \cdots \otimes \sigma_{p}$. Since $\Xi_{v}$ is irreducible for all $v[\mathrm{Ta}], \Xi$ is irreducible. Therefore, $\Pi=\Xi=$ Ind $\sigma_{1} \otimes \cdots \otimes \sigma_{p}$.

Suppose $\sigma_{1}$ is not self-contragredient. Then consider $L\left(s, \tilde{\sigma}_{1} \times \pi\right)$. It is holomorphic at $s=1$ since $\sigma_{1}$ is not self-contragredient (Ki6, Proposition 3.1] or C-Ki-PS-S, Proposition 3.1]). But $L\left(s, \tilde{\sigma}_{1} \times \pi\right)=\prod_{i=1}^{p} L\left(s, \tilde{\sigma}_{1} \times \sigma_{i}\right)$ has a pole at $s=1$. Contradiction.

Remark. It is expected that $L\left(s, \sigma_{i}, \wedge^{2}\right)$ has a pole at $s=1$ for all $i$ (hence $n_{i}$ is even Ki1]), and $\sigma_{i v_{0}}$ is supercuspidal. In fact, we prove in a separate paper Ki6 that the above theorem is true in general without supercuspidal component condition, and also that $L\left(s, \sigma_{i}, \wedge^{2}\right)$ has a pole at $s=1$ for all $i$. The only thing we need to show is that the Rankin-Selberg $L$-function $L(s, \sigma \times \pi)$ is holomorphic for Re $s>1$ for any cuspidal representation $\sigma$ of $G L_{m}\left(\mathbb{A}_{F}\right)$. The proof requires a deep result on the spherical unitary dual due to Barbasch and Moy [B-Mo]. Our purpose in this paper is to give a simpler proof of the above theorem without using unitary dual. Recently Ginzburg, Rallis and Soudry proved that the Rankin-Selberg $L$ function $L(s, \sigma \times \pi)$ is holomorphic for $\operatorname{Re} s>1$ for any cuspidal representation $\sigma$ of $G L_{m}\left(\mathbb{A}_{F}\right)$, and hence the above theorem without any condition. They can also obtain the backward lifting.

By combining Theorem 2.8 and Corollary 3 in section 6 of [C-Ki-PS-S], we obtain

Theorem 2.10. Let $\pi=\bigotimes_{v} \pi_{v}$ be a generic cuspidal representation of $S O_{2 n+1}\left(\mathbb{A}_{F}\right)$ which has one unramified tempered local component. Then the strong lift is of the form $\sigma_{1} \boxplus \cdots \boxplus \sigma_{p}$, where $\sigma_{i}$ is a cuspidal representation of $G L_{n_{i}}\left(\mathbb{A}_{F}\right)$.

In the next two corollaries, let $\pi=\bigotimes_{v} \pi_{v}$ be a generic cuspidal representation of $S O_{2 n+1}\left(\mathbb{A}_{F}\right)$ such that $\pi$ contains either a supercuspidal component, or tempered unramified component. 
Corollary 2.11. For any cuspidal representation $\sigma$ of $G L_{m}\left(\mathbb{A}_{F}\right)$, the RankinSelberg L-function $L(s, \sigma \times \pi)$ is holomorphic, except possibly at $s=0,1$. It is entire if $m>2 n$.

Proof. By Theorems 2.9 and 2.10, the strong lift $\Pi$ of $\pi$ is given by $\sigma_{1} \boxplus \cdots \boxplus \sigma_{p}$, where the $\sigma_{i}$ 's are cuspidal representations of $G L$. Hence

$$
L(s, \sigma \times \pi)=L(s, \sigma \times \Pi)=\prod_{i=1}^{p} L\left(s, \sigma \times \sigma_{i}\right) .
$$

Our assertion follows from the well-known fact on the Rankin-Selberg $L$-functions of $G L_{a} \times G L_{b}$.

Corollary 2.12. Suppose $\pi_{v}=$ Ind $|\operatorname{det}|^{r_{1}} \tau_{1} \otimes \cdots \otimes \mid$ det $\left.\right|^{r_{a}} \tau_{a} \otimes \tau_{0}$, where $0<r_{a} \leq$ $\cdots \leq r_{1}, \tau_{1}, \ldots, \tau_{p}$ are discrete series of $G L$, and $\tau_{0}$ is a tempered representation of $S O_{2 l+1}\left(F_{v}\right)$. This is the case due to standard module conjecture, proved by $\mathrm{Mu}$ Theorem 0.4]. Then $r_{1}<\frac{1}{2}$.

Proof. Let $\Pi=\bigotimes_{v} \Pi_{v}$ be the strong lift of $\pi$, which is of the form $\sigma_{1} \boxplus \cdots \boxplus \sigma_{p}$. In particular, $\Pi_{v}$ is generic and unitary. Now $\Pi_{v}$ is the unique quotient of

$$
\text { Ind }|\operatorname{det}|^{r_{1}} \tau_{1} \otimes \cdots \otimes|\operatorname{det}|^{r_{a}} \tau_{a} \otimes \Pi_{0} \otimes|\operatorname{det}|^{-r_{a}} \tilde{\tau}_{a} \otimes \cdots \otimes|\operatorname{det}|^{-r_{1}} \tilde{\tau}_{1},
$$

where $\Pi_{0}$ is the local lift of $\tau_{0}$. Our assertion follows from the classification of unitary dual of $G L_{n}$ Ta.

\section{REDUCIBILITY CRITERION}

In this section, let $k$ be a $p$-adic field of characteristic zero. Let $\tau$ be a generic supercuspidal representation of $S O_{2 n+1}(k)$. Let $\Pi$ be its lift to $G L_{2 n}(k)$. By Lemma 2.5 , it is of the form

$$
\Pi=\sigma_{1} \boxplus \cdots \boxplus \sigma_{p},
$$

where the $\sigma_{i}$ 's are (unitary) supercuspidal representations of $G L_{n_{i}}(k)$ and $L\left(s, \sigma_{i}, \wedge^{2}\right)$ has a pole at $s=0$. In particular, $n_{i}$ is even, and $\sigma_{i}$ 's are selfcontragredient and have the trivial central character.

Let $\sigma$ be a self-contragredient supercuspidal representation of $G L_{m}(k)$. Then there exists a unique $s_{0} \geq 0$ such that the induced representation Ind $|\operatorname{det}|^{s} \sigma \otimes \tau$ is reducible at $s=s_{0},-s_{0}$ and irreducible at all other points. The deep result of Shahidi [Sh1] is that $s_{0} \in\left\{0, \frac{1}{2}, 1\right\}$.

Definition 3.1. $(\sigma, \tau)$ satisfies $(C i)$ if Ind $|\operatorname{det}|^{s} \sigma \otimes \tau$ is reducible at $s=i$.

Note that if $l=0$, we set the convention that $\tau=1$. In that case, $(\sigma, 1)$ satisfies either $\left(C \frac{1}{2}\right)$ or $(C 0)$. We give a precise criterion of when $(\sigma, \tau)$ satisfies $(C i)$ in terms of the functorial lift of $\tau$. By [Sh1, Corollary 7.6],

(1) $(\sigma, \tau)$ satisfies $(C 1)$ if and only if $L(s, \sigma \times \tau)$ has a pole at $s=0$.

(2) $(\sigma, \tau)$ satisfies $\left(C \frac{1}{2}\right)$ if and only if $L\left(s, \sigma, S^{2} m^{2}\right)$ has a pole at $s=0$.

(3) $(\sigma, \tau)$ satisfies $(C 0)$ if and only if $L(s, \sigma \times \tau) L\left(s, \sigma, S^{2} m^{2}\right)$ is holomorphic at $s=0$. This means that $L(s, \sigma \times \tau)$ is holomorphic at $s=0$ but $L\left(s, \sigma, \wedge^{2}\right)$ has a pole at $s=0$.

We prove

Proposition 3.2. Let $\sigma, \tau$ be as above. Then

(1) $(\sigma, \tau)$ satisfies $(C 1)$ if and only if $\sigma \simeq \sigma_{i}$ for some $i$. 
(2) $(\sigma, \tau)$ satisfies $\left(C \frac{1}{2}\right)$ if and only if $L\left(s, \sigma, \wedge^{2}\right)$ is holomorphic at $s=0$.

(3) $(\sigma, \tau)$ satisfies $(C 0)$ if and only if $\sigma \neq \sigma_{i}$ for all $i$ and $L\left(s, \sigma, \wedge^{2}\right)$ has a pole at $s=0$.

Proof. (1) Since $\Pi=\sigma_{1} \boxplus \cdots \boxplus \sigma_{p}$,

$$
L(s, \sigma \times \tau)=L(s, \sigma \times \Pi)=\prod_{i=1}^{p} L\left(s, \sigma \times \sigma_{i}\right) .
$$

Note that the Rankin-Selberg $L$-function $L\left(s, \rho_{1} \times \rho_{2}\right)$ of $G L_{a} \times G L_{b}$, has a pole at $s=0$ if and only if $\rho_{1} \simeq \tilde{\rho}_{2}$, where $\rho_{1}, \rho_{2}$ are supercuspidal representations of $G L_{a}, G L_{b}$, resp. Hence our assertion follows.

(2) and (3) follows from the identity $L(s, \sigma \times \sigma)=L\left(s, \sigma, \operatorname{Sym}^{2}\right) L\left(s, \sigma, \wedge^{2}\right)$.

Corollary 3.3. Suppose $(\sigma, \tau)$ satisfies (C1). Then $L(s, \sigma \times \sigma)^{-1}$ divides $L(s, \sigma \times$ $\tau)^{-1}$ as polynomials in $q^{-s}$, namely,

$$
L(s, \sigma \times \tau)=L(s, \sigma \times \sigma) \prod_{j}\left(1-\alpha_{j} q^{-s}\right)^{-1},
$$

where $\alpha_{j} \in \mathbb{C}$ is of absolute value 1 .

Proof. It is clear from the equation in the above proof.

The following is expected (cf. [Ki6] Conjecture 8.3]).

Conjecture 3.4. Let $F$ be a number field and $\mathbb{A}_{F}$ its ring of adeles. Let $\tau=\bigotimes_{v} \tau_{v}$ be a generic cuspidal representation of $S O_{2 n+1}\left(\mathbb{A}_{F}\right)$ such that $\tau_{v_{0}}$ is supercuspidal. Suppose the lift of $\tau_{v_{0}}$ to $G L_{2 n}\left(F_{v_{0}}\right)$ is of the form $\sigma_{1 v_{0}} \boxplus \cdots \boxplus \sigma_{k v_{0}}$, where $\sigma_{i v_{0}}$ is a supercuspidal representation of $G L_{n_{i}}\left(F_{v_{0}}\right)$ such that $L\left(s, \sigma_{i v_{0}}, \wedge^{2}\right)$ has a pole at $s=0$. Then the lift of $\tau$ is of the form $\Pi=\sigma_{1} \boxplus \cdots \boxplus \sigma_{k}$, where the $\sigma_{i}$ 's are cuspidal representations of $G L_{n_{i}}\left(\mathbb{A}_{F}\right)$.

\section{Review of the local Langlands' CORRESPONDENCE FOR $G L_{n}$}

In this section we follow $\mathrm{Ku}$. Let $k$ be a $p$-adic field of characteristic zero. Let $\mathcal{G}_{k}(n)$ be the set of isomorphism classes of admissible representations of $W_{k} \times S L_{2}(\mathbb{C})$ of degree $n$, i.e., $\phi: W_{k} \times S L_{2}(\mathbb{C}) \longrightarrow G L_{n}(\mathbb{C})$. Here two representations into $G L_{n}(\mathbb{C})$ are said to be equivalent if they are conjugate by an element of $G L_{n}(\mathbb{C})$. We identify the element of the set $\mathcal{G}_{k}(1)$, the characters of $W_{k}$, with characters of $k^{\times}$via the reciprocity isomorphism $r_{k}: W_{k}^{a b} \longleftarrow k^{\times}$. Let $\mathcal{A}_{k}(n)$ be the set of inequivalent classes of admissible representations of $G L_{n}(k)$. The following theorem is called the local Langlands' correspondence for $G L_{n}$.

Theorem 4.1 ([[-T], $[\mathrm{He}])$. For each $n \geq 1$, there is a canonical bijection

$$
\pi_{k}: \mathcal{G}_{k}(n) \longrightarrow \mathcal{A}_{k}(n), \quad \rho \longmapsto \pi_{k}(\rho),
$$

such that

(1) $\pi_{k}(\rho(\chi))=\pi_{k}(\rho)(\chi)$ for any character $\chi$ of $k^{\times}$.

(2) $\operatorname{det}(\rho)$ corresponds to $\omega_{\pi_{k}(\rho)}$, the central character of $\pi_{k}(\rho)$ via the isomorphism of local class field theory.

(3) $\widetilde{\pi_{k}(\rho)}=\pi_{k}(\tilde{\rho})$.

(4) $L\left(s, \rho_{1} \otimes \rho_{2}\right)=L\left(s, \pi_{k}(\rho) \times \pi_{k}(\rho)\right)$.

(5) $\epsilon\left(s, \rho_{1} \otimes \rho_{2}, \psi\right)=\epsilon\left(s, \pi_{k}(\rho) \times \pi_{k}(\rho), \psi\right)$. 
(6) $\pi_{k}$ preserves conductors.

(7) If $K$ is a finite Galois extension of a field $k$, then $\pi_{K}$ is compatible with the natural actions of $\operatorname{Gal}(K / k)$ on $\mathcal{G}_{K}$ and $\mathcal{A}_{K}$.

Theory of Jordan normal forms implies that a unipotent matrix in $G L_{n}(\mathbb{C})$ is conjugate to $J\left(p_{1}\right) \oplus J\left(p_{2}\right) \oplus \cdots \oplus J\left(p_{s}\right), p_{1} \geq p_{2} \geq \cdots \geq p_{s}, p_{1}+p_{2}+\cdots+p_{s}=$ $n$, where $J(p)$ is the $p \times p$ Jordan matrix with entries 1 just above the diagonal and zero everywhere else. Therefore unipotent classes in $G L_{n}(\mathbb{C})$ are in 1 to 1 correspondence with partitions $\lambda$ of $N$. We use the following standard notation for $\lambda: \lambda=\left(1^{r_{1}}, 2^{r_{2}}, 3^{r_{3}}, \cdots\right)$, where $r_{j}$ is the number of $p_{i}$ equal to $j$.

We say that a unipotent element $u$ is distinguished in $G$ if all maximal tori of $\operatorname{Cent}(u, G)$ are contained in the center of $G^{\circ}$, the connected component of the identity. This is equivalent to the fact that the unipotent orbit $O$ (conjugacy classes) of $u$ does not meet any proper Levi subgroup of $G$. In the case of $G L_{n}(\mathbb{C})$, there is only one distinguished unipotent orbit, namely, the one attached to the partition $(n)$. (See [Ki5, section 3] for detail.)

Let $O$ be the distinguished unipotent orbit in $G L_{p}(\mathbb{C})$. There is a homomorphism $\phi_{2}: S L_{2}(\mathbb{C}) \longrightarrow G L_{p}(\mathbb{C})$, given by $O$. Given a homomorphism $\phi_{1}: W_{k} \longrightarrow$ $G L_{m}(\mathbb{C})$, which parametrizes a supercuspidal representation $\sigma$ of $G L_{m}(k)$, we attach a homomorphism

$$
\phi_{1} \otimes \phi_{2}: W_{k} \times S L_{2}(\mathbb{C}) \longrightarrow G L_{m p}(\mathbb{C}) .
$$

It parametrizes the Steinberg representation, denoted by $S t(\sigma, p)$. It is the unique subrepresentation of

$$
\text { Ind }|\operatorname{det}|^{\frac{p-1}{2}} \sigma \otimes|\operatorname{det}|^{\frac{p-1}{2}-1} \sigma \cdots \otimes|\operatorname{det}|^{-\frac{p-1}{2}} \sigma .
$$

We note that the unique quotient of the above induced representation is parametrized by

$$
\begin{aligned}
|\operatorname{det}|^{\frac{p-1}{2}} \phi_{1} \oplus|\operatorname{det}|^{\frac{p-1}{2}-1} \phi_{1} \oplus \cdots \oplus|\operatorname{det}|^{-\frac{p-1}{2}} \phi_{1}: \\
W_{k} \times S L_{2}(\mathbb{C}) \longrightarrow G L_{m}(\mathbb{C}) \times \cdots \times G L_{m}(\mathbb{C}) \subset G L_{m p}(\mathbb{C}) .
\end{aligned}
$$

\section{LOCAL LANGLANDS' CORRESPONDENCE FOR ODD-ORTHOGONAL GROUPS}

Recall the formulation of the local Langlands' correspondence for odd-orthogonal groups; Let $k$ be a $p$-adic field of characteristic zero and $G=S O_{2 n+1}(k)$. Then ${ }^{L} G^{\circ}=S p_{2 n}(\mathbb{C})$. Let $\phi: W_{k} \times S L_{2}(\mathbb{C}) \longrightarrow S p_{2 n}(\mathbb{C})$ be an admissible representation.

Then the local Langlands' correspondence predicts that $\phi$ parametrizes a finite set $\Pi_{\phi}$, called $L$-packet, of isomorphism classes of irreducible admissible representations of $G$, and every admissible representation of $G$ belongs to $\Pi_{\phi}$ for a unique $\phi$. Two elements $\pi, \pi^{\prime}$ in the same set $\Pi_{\phi}$ would have the same $L$ - and $\epsilon$-factors, and are hence called $L$-indistinguishable. We have $\mathrm{Bo}$

(1) the elements of $\Pi_{\phi}$ are square integrable if and only if $\operatorname{Im}(\phi)$ is not contained in any proper parabolic subgroup of $S p_{2 n}(\mathbb{C})$.

(2) the elements of $\Pi_{\phi}$ are tempered if and only if $\phi\left(W_{k}\right)$ is bounded.

The representations in the $L$-packet $\Pi_{\phi}$ are parametrized by the component group $C_{\phi}=S_{\phi} / Z_{L} G^{\circ} S_{\phi}^{\circ}$, where $S_{\phi}$ is the centralizer of $\operatorname{Im}(\phi)$ in ${ }^{L} G^{\circ}, S_{\phi}^{\circ}$ is the connected component of the identity, and $Z_{L} G^{\circ}$ is the center of ${ }^{L} G^{\circ}$.

Here we note that for $G=G L_{n}(\mathbb{C})$, the centralizer $Z_{G}(S)$ is connected for any subset $S$ of $G$. Hence the component group $C_{\phi}$ is trivial for any $\phi$. This justifies the 
formulation of the local Langlands' correspondence in the case of $G L_{n}$ in section 4 without the $L$-packets.

In this section, we parametrize all square integrable representations of $S O_{2 n+1}(k)$ with generic supercuspidal support by admissible homomorphisms of $W_{k} \times S L_{2}(\mathbb{C})$. We cannot construct all the elements of the $L$-packet $\Pi_{\phi}$ for a given admissible homomorphism $\phi$, since we expect that it should contain certain non-generic supercuspidal representations.

First we need the following: Let $\sigma$ be a self-dual supercuspidal representation of $G L_{2 n}(k)$ and let $\phi: W_{k} \longrightarrow G L_{2 n}(\mathbb{C})$ be a self-dual admissible homomorphism, corresponding to a supercuspidal representation $\sigma$. Let $L\left(s, \operatorname{Sym}^{2}(\phi)\right), L\left(s, \wedge^{2}(\phi)\right)$ be the Artin $L$-functions attached to the symmetric square and the exterior square map. More precisely, let $S y m^{2}\left(\right.$ resp. $\wedge^{2}$ ) be the finite dimensional representation

$$
g: X \longmapsto{ }^{t} g X g, \quad g \in G L_{2 n}(\mathbb{C}),
$$

of $G L_{2 n}(\mathbb{C})$ on the space of symmetric (resp. skew-symmetric) $2 n \times 2 n$-matrices. Then $L\left(s, \operatorname{Sym}^{2}(\phi)\right), L\left(s, \wedge^{2}(\phi)\right)$ are the Artin $L$-functions attached to $S_{y m}^{2} \circ$ $\phi, \wedge^{2} \circ \phi$, resp. Let $L\left(s, \sigma, S y m^{2}\right), L\left(s, \sigma, \wedge^{2}\right)$ be the Shahidi's $L$-functions given by the Langlands-Shahidi method [Sh1]. More precisely, $L\left(s, \sigma, S_{y m^{2}}\right)$ comes from the theory of Eisenstein series relative to $G L_{2 n} \subset S O_{4 n+1} ; L\left(s, \sigma, \wedge^{2}\right)$ comes from the theory of Eisenstein series relative to $G L_{2 n} \subset S O_{4 n}$.

Assumption 5.1. (1) $L\left(s, S^{2} m^{2}(\phi)\right)$ has a pole at $s=0$ if and only if $L\left(s, \sigma, S^{2} m^{2}\right)$ has a pole at $s=0$.

(2) $L\left(s, \wedge^{2}(\phi)\right)$ has a pole at $s=0$ if and only if $L\left(s, \sigma, \wedge^{2}\right)$ has a pole at $s=0$.

Remark. In $\mathrm{P}-\mathrm{R}$, Proposition 5.2], (2) is shown to be true for $n=2$. The above assumption is the same as the assertion that the Shahidi's $L$-factors are Artin $L$ factors. It is an observation due to F. Shahidi and it is true for any $L$-function as follows; Let $\sigma$ be an irreducible supercuspidal representation and $L\left(s, \sigma, r_{i}\right)$ be the Shahidi's $L$-function defined in [Sh1, section 7]. By [Sh1, Proposition 7.3], it is a product of $\left(1-\alpha q^{-s}\right)^{-1}$, where $\alpha \in \mathbb{C}$ is of absolute value one. Hence if $s_{0}$ is a pole of $L\left(s, \sigma, r_{i}\right)$, then there exists an unramified character $\chi$ such that $L\left(s, \sigma \otimes \chi, r_{i}\right)$ has a pole at $s=0$. Therefore, if Shahidi's $L$-function is the same as the Artin $L$-function at $s=0$ for any unitary supercuspidal representation, then so is the case everywhere else.

It may be much harder to prove that Shahidi's $\epsilon$-factors are Artin $\epsilon$-factors.

Lemma 5.2. Let $\phi: W_{k} \times S L_{2}(\mathbb{C}) \longrightarrow G L_{2 n}(\mathbb{C})$ be an admissible homomorphism, corresponding to an irreducible admissible representation $\sigma$. Under Assumption 5.1, $\phi$ factors through $S p_{2 n}(\mathbb{C})\left(O_{2 n}(\mathbb{C})\right.$, resp. $)$ if and only if $L\left(s, \sigma, \wedge^{2}\right)\left(L\left(s, \sigma, S_{y m}^{2}\right)\right.$, resp.) has a pole at $s=0$.

Proof. Note that $\phi$ factors through $S p_{2 n}(\mathbb{C})\left(O_{2 n}(\mathbb{C})\right.$, resp. $)$ if and only if the representation $\wedge^{2} \circ \phi\left(S y m^{2} \circ \phi\right.$, resp. $)$ contains the trivial representation. This is the case if and only if the Artin $L$-function $L\left(s, \wedge^{2}(\phi)\right)\left(L\left(s, \operatorname{Sym}^{2}(\phi)\right)\right.$, resp.) has a pole at $s=0$. Our result follows from Assumption 5.1 if $\sigma$ is supercuspidal. If $\sigma$ is arbitrary, it is a subquotient of an induced representation, induced from a supercuspidal representation. Hence by multiplicativity of $L$-factors, we can see easily that Shahidi's exterior square $L$-function $L\left(s, \sigma, \wedge^{2}\right)$ is the Artin $L$-function under Assumption 5.1. The same is true for the symmetric square $L$-function $L\left(s, \sigma, S_{y m}^{2}\right)$. 
From this section on, we make Assumption 5.1.

5.1 Parametrization for generic supercuspidal representations. Let $\tau$ be a generic supercuspidal representation of $S O_{2 n+1}(k)$. Let $\Pi$ be the lift of $\tau$. It is of the form $\rho_{1} \boxplus \cdots \boxplus \rho_{p}$, where the $\rho_{i}$ 's are supercuspidal representations of $G L_{2 n_{i}}(k)$ such that $L\left(s, \rho_{i}, \wedge^{2}\right)$ has a pole at $s=0$.

Let $\phi_{i}: W_{k} \longrightarrow G L_{2 n_{i}}(\mathbb{C})$ be the admissible homomorphism corresponding to $\rho_{i}$. Then it factors through $S p_{2 n_{i}}(\mathbb{C})$ by Lemma 5.2. Hence we have a homomorphism

$$
\phi=\phi_{1} \oplus \cdots \oplus \phi_{p}: W_{k} \longrightarrow S p_{2 n_{1}}(\mathbb{C}) \times \cdots \times S p_{2 n_{p}}(\mathbb{C}) \hookrightarrow S p_{2 n}(\mathbb{C}),
$$

which parametrizes the given generic supercuspidal representation $\tau$. We set $\phi$ to be trivial on $S L_{2}(\mathbb{C})$. Now, since $S p_{2 n}(\mathbb{C})$ is connected, $S_{\phi}$, the centralizer of $\operatorname{Im}(\phi)$ in ${ }^{L} G^{\circ}$, is connected. Hence $C_{\phi}=1$. Namely, the $L$-packet $\Pi_{\phi}$ consists of only one element $\tau$.

5.2 Parametrization for discrete series representations. Let us recall the recent classification of discrete series due to Moeglin and Tadic [M-Ta]. Let

$$
\pi=\underbrace{\sigma_{1} \otimes \cdots \otimes \sigma_{1}}_{u_{1}} \otimes \cdots \otimes \underbrace{\sigma_{t} \otimes \cdots \otimes \sigma_{t}}_{u_{t}} \otimes \tau
$$

be a generic supercuspidal representation of a Levi subgroup of $S O_{2 n+1}(k)$, where $\sigma_{1}, \ldots, \sigma_{t}$ are non-equivalent, self-contragredient supercuspidal representations of $G L_{m_{i}}(k)$ and $\tau$ is a generic supercuspidal representation of $S O_{2 l+1}(k)$.

(1) If $\left(\sigma_{i}, \tau\right)$ satisfies $(C 1)$, then we attach a distinguished unipotent orbit of $O_{2 u_{i}+1}(\mathbb{C})$.

(2) If $\left(\sigma_{i}, \tau\right)$ satisfies $\left(C \frac{1}{2}\right)$, then we attach a distinguished unipotent orbit of $S p_{2 u_{i}}(\mathbb{C})$.

(3) If $\left(\sigma_{i}, \tau\right)$ satisfies $(C 0)$, then we attach a distinguished unipotent orbit of $\mathrm{O}_{2 u_{i}}(\mathbb{C})\left(u_{i} \geq 2\right)$.

Note that a distinguished unipotent orbit of $S O_{2 n+1}(\mathbb{C}), O_{2 n}(\mathbb{C})$ is given by a partition $\left(p_{1}, \ldots, p_{r}\right)$, where the $p_{i}$ 's are distinct odd positive integers such that $p_{1}+\cdots+p_{r}=2 n+1$ or $2 n$. A distinguished unipotent orbit of $S p_{2 n}(\mathbb{C})$ is given by $\left(p_{1}, \ldots, p_{r}\right)$, where the $p_{i}$ 's are distinct even positive integers such that $p_{1}+\cdots+p_{r}=2 n$.

Given a distinguished unipotent orbit $O=\left(p_{1}, \ldots, p_{r}\right)$, we form a set $P(O)$ of ordered partitions as follows [M1: $\mathfrak{p}=\left(; p_{1}, \ldots, p_{r}\right) \in P(O)$ if and only if

(1) $\left(p_{1}, \ldots, p_{r}\right)$ is $O$ if we ignore the order.

(2) For all $1 \leq j \leq\left[\frac{r+1}{2}\right], p_{2 j-1}>p_{2 j}$ and there does not exist $1 \leq k \leq\left[\frac{r+1}{2}\right]$ such that $p_{2 j-1}>p_{2 k-1}>p_{2 j}>p_{2 k}$.

(3) If there exists a $1 \leq k \leq r$ such that $p_{2 j-1}>p_{k}>p_{2 j}$, then $k<2 j-1$.

We set $p_{r+1}=0$ if $r$ is odd.

Let $A(O)$ be a finite abelian group generated by the order two elements $a\left(p_{1}\right), \ldots$, $a\left(p_{r}\right)$. Let $\bar{A}(\mathfrak{p})=A(O) / K_{\mathfrak{p}}$, where $K_{\mathfrak{p}}$ is generated by $a\left(p_{2 i-1}\right) a\left(p_{2 i}\right)^{-1}$ for all $1 \leq i \leq\left[\frac{r+1}{2}\right]$. We set $a\left(p_{r+1}\right)=1$ if $r$ is odd. We note that $|\bar{A}(\mathfrak{p})|=2^{\left[\frac{r}{2}\right]}$.

Then (see [M1]) Springer $(O) \simeq \bigcup_{\mathfrak{p} \in P(O)} \widehat{\overline{A(p)}}$, where $\widehat{\bar{A}(\mathfrak{p})}$ is the character group of $\bar{A}(\mathfrak{p})$. We recall that the Springer correspondence is an injective map from the characters of $W$, the Weyl group of ${ }^{L} G^{\circ}$ into the set of pairs $(O, \eta)$, where $O$ is a unipotent orbit in ${ }^{L} G^{\circ}$ and $\eta$ is a character of $A(O)$. Given a unipotent orbit $O$ in ${ }^{L} G^{\circ}$, Springer $(O)$ is the set of characters of $A(O)$ which are in the image 
of the Springer correspondence. Also recall that if $O$ is a unipotent orbit in ${ }^{L} G^{\circ}$, $A(O)=C(u) / C(u)^{0}$, where $C(u)=\operatorname{Cent}\left(u,{ }^{L} G^{\circ}\right), u \in O$.

Let $\mathfrak{p}=\left(; p_{1}, \ldots, p_{r}\right) \in P(O)$. Suppose $r$ is odd, and write

$$
\mathfrak{p}=\left(; a_{1}, b_{1}, \ldots, a_{s}, b_{s}, a_{s+1}\right) .
$$

Then we can form a chain

$$
\begin{aligned}
\lambda_{\mathfrak{p}}= & \left(\frac{a_{1}-1}{2}, \frac{a_{1}-3}{2}, \ldots,-\frac{b_{1}-1}{2}, \ldots, \frac{a_{s}-1}{2}, \frac{a_{s}-3}{2}, \ldots,\right. \\
& \left.-\frac{b_{s}-1}{2}, \frac{a_{s+1}-1}{2}, \frac{a_{s+1}-3}{2}, \ldots, \frac{a_{s+1}+1}{2}-\left[\frac{a_{s+1}}{2}\right]\right) .
\end{aligned}
$$

Notice that

$$
\frac{a_{s+1}+1}{2}-\left[\frac{a_{s+1}}{2}\right]= \begin{cases}\frac{1}{2}, & \text { if } a_{s+1} \text { is even, } \\ 1, & \text { if } a_{s+1} \text { is odd. }\end{cases}
$$

Note that if $\mathfrak{p}=(; a, b)$ and $\pi=\sigma \otimes \cdots \otimes \sigma$, where $\sigma$ is a supercuspidal representation of $G L$, the following induced representation of $G L$,

$$
\operatorname{Ind} d^{G L} \lambda_{\mathfrak{p}} \otimes \pi=\operatorname{Ind}|\operatorname{det}|^{\frac{a-1}{2}} \sigma \otimes|\operatorname{det}|^{\frac{a-3}{2}} \sigma \otimes \cdots \otimes|\operatorname{det}|^{-\frac{b-1}{2}} \sigma,
$$

has a unique subrepresentation, which is $|\operatorname{det}|^{\frac{a-b}{4}} \operatorname{St}\left(\sigma, \frac{a+b}{2}\right)$.

We first consider a special case when $\pi=\sigma \otimes \cdots \otimes \sigma \otimes \tau$. Let $O$ be a distinguished unipotent orbit, determined by $(\sigma, \tau)$. We write $\mathfrak{p} \in P(O)$ as $\mathfrak{p}=$ $\left(; a_{1}, b_{1}, \ldots, a_{s}, b_{s}, a_{s+1}\right)$. For simplicity, assume that $a_{s+1}$ is even. By inducing in stages, we see that $\operatorname{Ind}^{G} \lambda_{\mathfrak{p}} \otimes \pi$ has a subrepresentation, namely,

$$
\begin{aligned}
\operatorname{Ind}^{G}|\operatorname{det}|^{\frac{a_{1}-b_{1}}{4}} \operatorname{St}\left(\sigma, \frac{a_{1}+b_{1}}{2}\right) & \otimes \cdots \otimes|\operatorname{det}|^{\frac{a_{s}-b_{s}}{4}} \operatorname{St}\left(\sigma, \frac{a_{s}+b_{s}}{2}\right) \\
& \otimes|\operatorname{det}|^{\frac{a_{s+1}}{4}} \operatorname{St}\left(\sigma, \frac{a_{s+1}}{2}\right) \otimes \tau .
\end{aligned}
$$

Let $\operatorname{Unip}(\mathfrak{p})$ be the set of direct summands of the maximal completely reducible subrepresentation of the above induced representation. It is parametrized by $\widehat{\bar{A}(\mathfrak{p})}$. Let $\operatorname{Unip}(O)$ be the union of $\operatorname{Unip}(\mathfrak{p})$ as $\mathfrak{p}$ runs through $P(O)$. Then it is parametrized by Springer $(O)$. The result of Moeglin-Tadic [M-Ta is that they are all nonsupercuspidal square integrable representations with supercuspidal support $(\sigma, \tau)$. More generally, if $\pi=\underbrace{\sigma_{1} \otimes \cdots \otimes \sigma_{1}}_{u_{1}} \otimes \cdots \otimes \underbrace{\sigma_{t} \otimes \cdots \otimes \sigma_{t}}_{u_{t}} \otimes \tau$, the non-supercuspidal square integrable representations with supercuspidal support $\pi$, are parametrized by Springer $\left(O_{1}\right) \times \cdots \times \operatorname{Springer}\left(O_{t}\right)$, where the $O_{i}$ 's are attached to $\left(\sigma_{i}, \tau\right)$. In summary,

Theorem 5.3 ([M-Ta $]$ ). Let

$$
\pi=\underbrace{\sigma_{1} \otimes \cdots \otimes \sigma_{1}}_{u_{1}} \otimes \cdots \otimes \underbrace{\sigma_{t} \otimes \cdots \otimes \sigma_{t}}_{u_{t}} \otimes \tau
$$

be a generic supercuspidal representation of a Levi subgroup of G. All non-supercuspidal square integrable representations with supercuspidal support $\pi$ are parametrized by $\left(O_{1}, \ldots, O_{t}, \eta\right)$, where $O_{i}$ is a distinguished unipotent orbit determined by $\left(\sigma_{i}, \tau\right)$, and $\eta \in \operatorname{Springer}\left(O_{1}\right) \times \cdots \times \operatorname{Springer}\left(O_{t}\right)$. 
In this paper, for simplicity, we restrict ourselves to the special case when $\pi=\underbrace{\sigma \otimes \cdots \otimes \sigma}_{u} \otimes \tau$, where $\sigma$ is a supercuspidal representation of $G L_{m}(k)$ and $\tau$ is a generic supercuspidal representation of $S O_{2 l+1}(k)$, and $O$ be a distinguished unipotent orbit, determined by $(\sigma, \tau)$. Hence $m u+l=n$. All the square integrable representations parametrized by the unipotent orbit $O$ will be in the same $L$-packet. We now give parametrization:

Given a positive integer $p \in O$, we have a discrete series of $G L_{m p}(k)$, given as the subrepresentation of $|\operatorname{det}|^{\frac{p-1}{2}} \sigma \otimes|\operatorname{det}|^{\frac{p-1}{2}-1} \otimes \cdots \otimes|\operatorname{det}|^{-\frac{p-1}{2}} \sigma$. We denoted it by $S t(\sigma, p)$ in section 4 . Let $\phi_{p}: W_{k} \times S L_{2}(\mathbb{C}) \longrightarrow G L_{m p}(\mathbb{C})$ be the homomorphism attached to the discrete series $S t(\sigma, p)$.

Lemma 5.4. $\phi_{p}$ factors through $S p_{m p}(\mathbb{C})$.

Proof. First of all, we emphasize that $m p$ is even. It will be clear in the proof. We need to show that $L\left(s, S t(\sigma, p), \wedge^{2}\right)$ has a pole at $s=0$. If $(\sigma, \tau)$ satisfies either $(C 1)$ or $(C 0)$, then $p$ is odd and $m$ is even. By looking at [Sh5, Proposition 8.1], the $L$-function contains the factor $L\left(s, \sigma, \wedge^{2}\right)$, which has a pole at $s=0$.

Suppose $(\sigma, \tau)$ satisfies $\left(C \frac{1}{2}\right)$. Then $p$ is even. Then the $L$-function contains the factor $L\left(s, \sigma, S^{2} m^{2}\right)$, which has a pole at $s=0$.

First consider the case when $(\sigma, \tau)$ satisfies $(C 1)$. Then the lift of $\tau$ is given by Ind $\sigma \otimes \sigma_{1} \otimes \cdots \sigma_{a}$ by Proposition 3.2. Hence $m$ is even. Let $O=\left(p_{1}, \ldots, p_{r}\right)$ be a distinguished unipotent orbit in $S O_{2 u+1}(\mathbb{C})$. Hence $p_{1}+\cdots+p_{r}=2 u+1$. Then $p_{i}$ and $r$ are odd. In this case, Let

$$
\phi_{0}: W_{k} \longrightarrow S p_{2 l-m}(\mathbb{C})
$$

be the homomorphism which parametrizes Ind $\sigma_{1} \otimes \cdots \otimes \sigma_{a}$. Let

$$
\phi_{i}: W_{k} \times S L_{2}(\mathbb{C}) \longrightarrow S p_{m p_{i}}(\mathbb{C}),
$$

be the homomorphism which parametrizes $S t\left(\sigma, p_{i}\right)$. Then

$$
\begin{aligned}
\phi_{0} \oplus \phi_{1} \oplus \cdots \oplus \phi_{r}: W_{k} \times S L_{2}(\mathbb{C}) & \longrightarrow S p_{2 l-m}(\mathbb{C}) \times S p_{m p_{1}}(\mathbb{C}) \times \cdots \times S p_{m p_{r}}(\mathbb{C}) \\
& \hookrightarrow S p_{2 n}(\mathbb{C}),
\end{aligned}
$$

parametrizes the discrete series attached to $(\sigma, \tau)$ and $O$.

Next, let $(\sigma, \tau)$ satisfy $\left(C \frac{1}{2}\right)$. In this case, $m$ may not be even. Let $O=\left(p_{1}, \ldots, p_{r}\right)$ be a distinguished unipotent orbit of $S p_{2 u}(\mathbb{C})$ with $p_{1}+\cdots+p_{r}=2 u$. Then $p_{i}$ is even. Let

$$
\phi_{0}: W_{k} \longrightarrow S p_{2 l}(\mathbb{C})
$$

be the homomorphism which parametrizes $\tau$ as in $\S 5.1$.

For each $p_{i} \in O$, let

$$
\phi_{i}: W_{k} \times S L_{2}(\mathbb{C}) \longrightarrow S p_{m p_{i}}(\mathbb{C}),
$$

be the homomorphism which parametrizes $S t\left(\sigma, p_{i}\right)$. Then

$$
\begin{aligned}
\phi_{0} \oplus \cdots \oplus \phi_{r}: W_{k} \times S L_{2}(\mathbb{C}) & \longrightarrow S p_{2 l}(\mathbb{C}) \times S p_{m p_{1}}(\mathbb{C}) \times \cdots \times S p_{m p_{r}}(\mathbb{C}) \\
& \hookrightarrow S p_{2 n}(\mathbb{C})
\end{aligned}
$$

parametrizes the discrete series attached to $(\sigma, \tau)$ and $O$.

Finally, let $(\sigma, \tau)$ satisfy $(C 0)$. By Proposition $3.2, L\left(s, \sigma, \wedge^{2}\right)$ has a pole at $s=0$. Hence $m$ is even. Let $O=\left(p_{1}, \ldots, p_{r}\right)$ be a distinguished unipotent orbit in $O_{2 u}(\mathbb{C})$ 
with $p_{1}+\cdots+p_{r}=2 u$. Then $p_{i}$ is odd. The parametrization is exactly the same as in $\left(C \frac{1}{2}\right)$ case.

Note that $C_{\phi} \simeq(\mathbb{Z} / 2 \mathbb{Z})^{r}$. Hence there are $2^{r}$ representations in the $L$-packet attached to the distinguished unipotent orbit $O=\left(p_{1}, \ldots, p_{r}\right)$. But the discrete series in the $L$-packet are parametrized by the subset $\operatorname{Springer}(O) \subset C_{\phi}$ whose cardinality is $\mid$ Springer $(O) \mid={ }_{r} C_{\left[\frac{r}{2}\right]}$. (See [Ki5, section 3] for more details.) A conjecture might be that the remaining representations in the $L$-packet are non-generic supercuspidal representations and non-supercuspidal square integrable representations with non-generic supercuspidal support.

\section{Functorial Lift From $S O_{5}$ to $G L_{5}$}

Let $F$ be a number field and $\mathbb{A}_{F}$ its ring of adeles. Let $\pi$ be a generic cuspidal representation of $S O_{5}\left(\mathbb{A}_{F}\right)$. Let $\Pi$ be a strong lift of $\pi$ to $G L_{4}\left(\mathbb{A}_{F}\right)$. Then we prove in [Ki6] that $\Pi$ is either cuspidal, or of the form $\sigma_{1} \boxplus \sigma_{2}$, where $\sigma_{i}$ 's are inequivalent self-contragredient cuspidal representations of $G L_{2}\left(\mathbb{A}_{F}\right)$. We also prove that if $\Pi$ is cuspidal, then $L\left(s, \Pi, \wedge^{2}\right)$ has a pole at $s=1$, and if $\Pi=\sigma_{1} \boxplus \sigma_{2}$, then $\sigma_{i}$ 's have the trivial central character. The proof uses the integral representation technique due to Ginzburg-Rallis-Soudry G-R-S]. However, in the following special case, we can prove the following proposition without the integral representation technique.

Proposition 6.1. Suppose $\pi_{v_{0}}$ is supercuspidal and $\Pi$ is of the form $\sigma_{1} \boxplus \sigma_{2}$. Then the $\sigma_{i}$ 's have the trivial central character and $\sigma_{i v_{0}}, i=1,2$, is supercuspidal.

Proof. By Lemma 2.5, $\Pi_{v_{0}}$ has to be either supercuspidal, or of the form $\rho_{1} \boxplus \rho_{2}$, where $\rho_{i}$ is a supercuspidal representation of $G L_{2}\left(F_{v_{0}}\right)$ with the trivial central character. Since $\Pi$ is not cuspidal, $\Pi_{v_{0}}$ cannot be supercuspidal. Hence $\sigma_{i v_{0}}=\rho_{i}$, $i=1,2$.

Suppose that the central character of $\sigma_{i}$ is not trivial. Since $\sigma_{i}$ is self-contragredient, it is monomial. Hence $L\left(s, \sigma_{i}, S y m^{2}\right)$ has a pole at $s=1$. Consider the situation $M=G L_{2} \subset S O_{5}$, and the global induced representation $I n d_{G L_{2}}^{S O_{5}}|\operatorname{det}|^{\frac{s}{2}} \sigma_{i}$. Since $L\left(s, \sigma_{i}, S y m^{2}\right)$ has a pole at $s=1,\left(M, \sigma_{i}\right)$ contributes to the residual (see spectrum Ki2 for details) and the residual automorphic representation is the (global) Langlands' quotient of $I n d_{G L_{2}}^{S O_{5}}|\operatorname{det}|^{\frac{s}{2}} \sigma_{i}$, and it is unitary. In particular, at $v=v_{0}$, the induced representation $\operatorname{Ind}_{G L_{2}}^{S O_{5}}|\operatorname{det}|^{\frac{s}{2}} \sigma_{i v_{0}}$ is reducible at $s=1$, because otherwise it cannot be unitary. Hence by reducibility criterion [Ca-Sh, $L\left(s, \rho_{i}, S_{y m^{2}}\right)$ has a pole at $s=0$. It means that by the identity $L\left(s, \rho_{i} \times \rho_{i}\right)=L\left(s, \rho_{i}, \operatorname{Sym}^{2}\right) L\left(s, \rho_{i}, \wedge^{2}\right)$, $L\left(s, \rho_{i}, \wedge^{2}\right)$ does not have a pole at $s=0$. It contradicts Lemma 2.5.

Remark. To put it in another way, we have shown that a self-contragredient monomial cuspidal representation of $G L_{2}(\mathbb{A})$ cannot have a supercuspidal component which has the trivial central character.

Remark. In an unpublished note, Jacquet, Piatetski-Shapiro and Shalika established a lift from $G S p_{4}$ to $G L_{4}$ and gave a criterion for the image of the lift. Since $S_{5} \simeq \mathrm{PGSp}_{4}$, our result is a very special case.

The last part of the proof of Proposition 6.1 is quite general. We record it as

Proposition 6.2. Let $P=M N$ be a maximal parabolic subgroup of a quasi-split group $G$, defined over a number field $F$ and $\sigma=\bigotimes_{v} \sigma_{v}$ be a cuspidal representation of $M(\mathbb{A})$ such that $\sigma_{v_{0}}$ is supercuspidal. If the induced representation $I\left(s, \sigma_{v_{0}}\right)=$ 
$\operatorname{Ind} d_{P}^{G} \sigma_{v_{0}} \otimes \exp ^{s \tilde{\alpha}, H_{P}()}$ is irreducible at $s=1$, then the Eisenstein series attached to $(M, \sigma)$ is holomorphic for $s>\frac{1}{2}$, especially at $s=1$.

Proof. Suppose the Eisenstein series attached to $(M, \sigma)$ has a pole at $s=s_{0}>\frac{1}{2}$. Then the residue at $s=s_{0}$ spans a residual automorphic representation $\bigotimes_{v} J\left(s_{0}, \sigma_{v}\right)$, where $J\left(s_{0}, \sigma_{v}\right)$ is the unique quotient of $I\left(s_{0}, \sigma_{v}\right)$. In particular, it is unitary. Since $\sigma_{v_{0}}$ is supercuspidal, the only reducibility points for $I\left(s, \sigma_{v_{0}}\right)$ for Re $s \geq 0$ are $\left\{0, \frac{1}{2}, 1\right\}$. Hence if $I\left(s, \sigma_{v_{0}}\right)$ is irreducible at $s=1$, it cannot be unitary at least for $s>\frac{1}{2}$. We obtain a contradiction.

Corollary 6.3. Let $\sigma=\bigotimes_{v} \sigma_{v}$ be a cuspidal representation of $G L_{n} \subset S O_{2 n+1}$ such that $\sigma_{v_{0}}$ is supercuspidal. If $I\left(s, \sigma_{v_{0}}\right)=$ Ind $d_{G L_{n}}^{S O_{2 n+1}} \sigma_{v_{0}} \mid$ det $\left.\right|^{\frac{s}{2}}$ is irreducible at $s=1$ (this is equivalent to the fact that $I\left(0, \sigma_{v_{0}}\right)$ is reducible), then $L\left(s, \sigma, S_{S^{2}}^{2}\right)$ is entire.

Proof. Apply Proposition 6.2 to $M=G L_{n} \subset G=S O_{2 n+1}$. Then the constant term of the Eisenstein series contains only one $L$-function $L\left(s, \sigma, S y m^{2}\right)$. Hence the only reducibility points for $I\left(s, \sigma_{v_{0}}\right)$ are $\{0,1\}$. Hence if $I\left(s, \sigma_{v_{0}}\right)$ is irreducible at $s=1$, then $I\left(s, \sigma_{v_{0}}\right)$ is irreducible for $s>0$ and not unitary. Hence the Eisenstein series attached to $(M, \sigma)$ is holomorphic for $s>0$. By following [Ki2, Theorem 3.1 , we can see that $L\left(s, \sigma, S y m^{2}\right)$ is entire.

Let $\pi$ be a generic cuspidal representation of $S O_{5}\left(\mathbb{A}_{F}\right)$, and let $\Pi$ be its lift to $G L_{4}\left(\mathbb{A}_{F}\right)$. Let $r_{5}: S p_{4}(\mathbb{C}) \longrightarrow G L_{5}(\mathbb{C})$ be the 5 dimensional representation, corresponding to the second fundamental weight. It is given by

$$
\operatorname{diag}\left(a, b, b^{-1}, a^{-1}\right) \longmapsto \operatorname{diag}\left(a b, a b^{-1}, 1, b a^{-1}, b^{-1} a^{-1}\right) .
$$

Under the identification $S O_{5} \simeq P G S p_{4}, L\left(s, \pi, r_{5}\right)$ appears in the constant term of Eisenstein series for $G S p_{6}\left(C_{3}-1\right.$ case of [Sh3]). More precisely, let $M=$ $G L_{1} \times G S p_{4}$ be a maximal Levi subgroup of $G S p_{6}$. Then by considering $\pi$ as a cuspidal representation of $M(\mathbb{A})$, we obtain $L\left(s, \pi, r_{5}\right)$ as a normalizing factor in the constant term of the Eisenstein series attached to $(M, \pi)$. Hence

Proposition 6.4 ([Sh3] $). L\left(s, \pi, r_{5}\right)$ has meromorphic continuation and satisfies the standard functional equation. It has no zeros for Re $s=1$.

Consider the composition of two maps

$$
S p_{4}(\mathbb{C}) \hookrightarrow G L_{4}(\mathbb{C}) \longrightarrow G L_{6}(\mathbb{C}) .
$$

Then by a well-known identity,

$$
\wedge^{2} \circ i=r_{5} \oplus 1 .
$$

Hence if $\Pi$ is cuspidal, $L\left(s, \Pi, \wedge^{2}\right)=L\left(s, \pi, r_{5}\right) L(s, 1)$. If $\Pi=\sigma_{1} \boxplus \sigma_{2}$, where $\sigma_{1}, \sigma_{2}$ are cuspidal representations of $G L_{2}\left(\mathbb{A}_{F}\right)$ with the trivial central character, then $L\left(s, \Pi, \wedge^{2}\right)=L\left(s, \sigma_{1} \times \sigma_{2}\right) L(s, 1) L(s, 1)$. Hence

By Proposition 6.4, we have

Proposition 6.5. Suppose $\Pi$ is cuspidal. Then $L\left(s, \Pi, \wedge^{2}\right)$ has a pole at $s=1$ and $L\left(s, \pi, r_{5}\right)$ is holomorphic at $s=1$. If $\Pi=\sigma_{1} \boxplus \sigma_{2}$, then $L\left(s, \Pi, \wedge^{2}\right)$ has a double pole at $s=1$, and hence $L\left(s, \pi, r_{5}\right)$ has a pole at $s=1$. 
Consider the exterior square lift $\wedge^{2} \Pi[$ Ki4]. First, we assume that $\Pi$ is cuspidal. Then by the main result in [Ki4, $\wedge^{2} \Pi$ is an automorphic representation of $G L_{6}\left(\mathbb{A}_{F}\right)$, which is of the form $\pi_{1} \boxplus \cdots \boxplus \pi_{k}$, where the $\pi_{i}$ 's are (unitary) cuspidal representations of $G L_{n_{i}}(\mathbb{A})$. Since $L\left(s, \Pi, \wedge^{2}\right)$ has a pole at $s=1$,

$$
\wedge^{2} \Pi=\tau \boxplus 1,
$$

where $\tau$ is an automorphic representation of $G L_{5}\left(\mathbb{A}_{F}\right)$ which is a functorial lift corresponding to $r_{5}$.

Suppose $\Pi=\sigma_{1} \boxplus \sigma_{2}$, where $\sigma_{i}$ 's are inequivalent self-contragredient cuspidal representations of $G L_{2}\left(\mathbb{A}_{F}\right)$ with the trivial central character. Then

$$
\wedge^{2} \Pi=\left(\sigma_{1} \otimes \sigma_{2}\right) \boxplus 1 \boxplus 1,
$$

where $\sigma_{1} \otimes \sigma_{2}$ is the functorial product whose existence was proved in [Ra]. (See Ki4 for a different proof.) It is an automorphic representation of $G L_{4}\left(\mathbb{A}_{F}\right)$. Hence $\tau=\left(\sigma_{1} \otimes \sigma_{2}\right) \boxplus 1$.

In conclusion, we have shown

Theorem 6.6. There exists a functional lift from cuspidal representations of

$$
S_{5}(\mathbb{A}) \simeq P G S p_{4}(\mathbb{A})
$$

to automorphic representations of $G L_{5}(\mathbb{A})$, corresponding to $r_{5}$.

\section{REFERENCES}

[B-Mo] D. Barbasch and A. Moy, Unitary spherical spectrum for p-adic classical groups, Acta Appl. Math. 44 (1996), 3-37. MR 98k:22067.

[Bo] A. Borel, Automorphic L-functions, Proc. Sympos. Pure Math. 33, part 2 (1979), 27-61. MR 81m:11056

[Ca-Sh] W. Casselman and F. Shahidi, On irreducibility of standard modules for generic representations, Ann. Sci. École Norm. Sup. 31 (1998), 561-589. MR 99f:22028

[C-Ki-PS-S] J. Cogdell, H. Kim, I.I. Piatetski-Shapiro, and F. Shahidi, On lifting from classical groups to $G L_{N}$, Publ. Math. IHES 93 (2001), 5-30.

[Co-PS] J. Cogdell and I.I. Piatetski-Shapiro, Converse theorems for $\mathrm{GL}_{n}$, Publ. Math. IHES 79 (1994), 157-214. MR 95m:22009

[G-R-S] D. Ginzburg, S. Rallis and D. Soudry, Self-dual automorphic GL $L_{n}$ modules and construction of a backward lifting from $G L_{n}$ to classical groups, IMRN 14 (1997). MR 98e:22013

[Go] D. Goldberg, R-groups and elliptic representations for similitude groups, Math. Ann. 307 (1997), 569-588. MR 98i:22024

[H-T] M. Harris and R. Taylor, On the geometry and cohomology of some simple Shimura varieties, preprint, 1998.

[He] G. Henniart, Une preuve simple des conjectures de Langlands pour $G L(n)$ sur un corps p-adique, Inv. Math. 139 (2000), 439-455. MR 2001e:11052

[J-PS-S] H. Jacquet, I.I. Piatetski-Shapiro and J. Shalika, Rankin-Selberg convolutions, Amer. J. Math. 105 (1983), 367-464. MR 85g:11044

[J-S] On Euler products and the classification of automorphic forms I,II, Amer. J. Math. 103 (1981), 499-558; 777-815. MR 82m:10050b

[Ja1] C. Jantzen, On supports of induced representations for symplectic and odd-orthogonal groups, Amer. J. Math. 119 (1997), 1213-1262. MR 99b:22028

[Ja2] $\quad$ On square-integrable representations of classical p-adic groups, Can. J. Math. 52 (2000), 539-581. MR 2001f:22056

[Ki1] H. Kim, Langlands-Shahidi method and poles of automorphic L-functions: application to exterior square L-functions, Can. J. Math. 51 (1999), 835-849. MR 2000f: 11058

[Ki2] Langlands-Shahidi method and poles of automorphic L-functions II, Israel J. Math. 117 (2000), 261-284. MR 2001i:11059a 

functions II, Israel J. Math. 118 (2000), 379. MR 2001i:11059b

[Ki4] - Functoriality for exterior square of $G L_{4}$ and symmetric fourth of $G L_{2}$, submitted.

[Ki5] _ Residual spectrum of split classical groups; contribution from Borel subgroups, Pacific J. Math. 199 (2001), 417-445.

[Ki6] Lesidual spectrum of odd-orthogonal groups, IMRN 17 (2001), 873-906. CMP 2002:02

[Ki7] _ On local L-functions and normalized intertwining operators, preprint.

$[\mathrm{Ku}] \quad \mathrm{S}$. Kudla, The local Langlands correspondence: the non-archimedean case, in Proceedings of Symposia in Pure Mathematics, vol. 55, part 2, American Mathematical Society, 1994, pp. 365-391. MR 95d:11065

[La1] R.P. Langlands, Problems in the theory of automorphic forms, Lecture Notes in Math., vol. 170, Springer-Verlag, Berlin-Heidelberg-New York, 1970, pp. 18-86. MR 46:1758

[La2] On the classification of irreducible representations of real algebraic groups, in Representation Theory and Harmonic Analysis on Semisimple Lie groups (P.J. Sally, Jr. and D.A. Vogan, ed.), Mathematical Surveys and Monographs, vol. 31, AMS, 1989, pp. 101-170. MR 91e:22017

[La3] - On the notion of an automorphic representation, Proc. Symp. Pure Math. 33, part 1, 1979, pp. 189-207. MR 81m:10055

[Li] J-S. Li, Some results on unramified principal series of p-adic groups, Math. Ann. 292 (1992), 747-761. MR 93d:22023

[M1] C. Moeglin, Représentations unipotentes et formes automorphes de carré intégrable, Forum mathematicum 6 (1994), 651-744. MR 95k:22024

[M2] Orbites unipotentes et spectre discret non ramifie, Le cas des groupes classiques déployś, Comp. Math. 77 (1991), 1-54. MR 92d:11054

[M3] _ Une conjecture sur le spectre résiduel des groupes classiques, preprint, 1994.

[M-W1] C. Moeglin and J.L. Waldspurger, Spectral Decomposition and Eisenstein series, une paraphrase de l'Ecriture, Cambridge tracts in mathematics, vol 113, Cambridge University Press, 1995. MR 97d:11083

[M-W2] C. Moeglin and J.L. Waldspurger, Le spectre résiduel de $G L(n)$, Ann. Scient. Éc. Norm. Sup. 22 (1989), 605-674. MR 91b:22028

[M-Ta] C. Moeglin and M. Tadic, Construction of discrete series for classical p-adic groups, preprint.

$[\mathrm{Mu}] \quad$ G. Muić, Some results on square integrable representations; irreducibility of standard representations, IMRN 14 (1998), 705-726. MR 99f:22031

[P-R] D. Prasad and D. Ramakrishnan, On the global root numbers of $G L(n) \times G L(m)$, Proc. Symp. in Pure Math. 66, 1999, pp. 311-330. MR 2000f:11060

[Ra] D. Ramakrishnan, Modularity of the Rankin-Selberg L-series, and multiplicity one for $S L(2)$, Ann. of Math. 152 (2000), 45-111. MR 2001g:11077]

[Ro] J. Rogawski, Representations of $G L(n)$ and division algebras over a p-adic field, Duke Math. J. 50 (1983), 161-196. MR 84j:12018

[Sh1] F. Shahidi, A proof of Langlands conjecture on Plancherel measures; complementary series for p-adic groups, Annals of Math. 132 (1990), 273-330. MR 91m:11095

[Sh2] _ On certain L-functions, Amer. J. Math 103 (1981), 297-355. MR 82i:11030

[Sh3] On the Ramanujan conjecture and finiteness of poles for certain L-functions, Ann. of Math. 127 (1988), 547-584. MR 89h:11021

[Sh4] - Twisted endoscopy and reducibility of induced representations for $p$-adic groups, Duke Math. J. 66, No. 1 (1992), 1-41. MR 93b:22034

[Sh5] _ On multiplicativity of local factors, In: Festschrift in honor of I.I. PiatetskiShapiro, Part II, Israel Math. Conf. Proc. 3, Weizmann, Jerusalem, 1990, pp. 279-289. MR 93e:11144

[Sh6] Local coefficients as Artin factors for real groups, Duke Math. J. 52 (1985), 973-1007. MR 87m:11049

[Ta] M. Tadic, Classification of unitary representations in irreducible representations of general linear group (non-Archimedean case), Ann. Sci. Ec. Norm. Sup. 19 (1986), 335-382. MR 88b:22021 
[Yo] H. Yoshida, On the unitarizability of principal series representations of $p$-adic Chevalley groups, J. Math. Kyoto Univ. 32, No 1 (1992), 155-233. MR 93c:22035

[Ze] A. Zelevinsky, Induced representations of reductive p-adic groups. II, Ann. Sci. Ec. Norm. Sup. 13 (1980), 165-210. MR 83g:22012

Department of Mathematics, University of Toronto, Toronto, ON M5S 3G3, Canada

E-mail address: henrykim@math.toronto.edu 